\title{
A Correspondence between Two Approaches to Interprocedural Analysis in the Presence of Join
}

\author{
Ravi Mangal $^{1}$, Mayur Naik ${ }^{1}$, and Hongseok Yang ${ }^{2}$ \\ ${ }^{1}$ Georgia Institute of Technology $\quad{ }^{2}$ University of Oxford
}

\begin{abstract}
Many interprocedural static analyses perform a lossy join for reasons of termination or efficiency. We study the relationship between two predominant approaches to interprocedural analysis, the summarybased (or functional) approach and the call-strings (or $k$-CFA) approach, in the presence of a lossy join. Despite the use of radically different ways to distinguish procedure contexts by these two approaches, we prove that post-processing their results using a form of garbage collection renders them equivalent. Our result extends the classic result by Sharir and Pnueli that showed the equivalence between these two approaches in the setting of distributive analysis, wherein the join is lossless.

We also empirically compare these two approaches by applying them to a pointer analysis that performs a lossy join. Our experiments on ten Java programs of size $400 \mathrm{~K}-900 \mathrm{~K}$ bytecodes show that the summary-based approach outperforms an optimized implementation of the $k$-CFA approach: the $k$-CFA implementation does not scale beyond $k=2$, while the summary-based approach proves up to $46 \%$ more pointer analysis client queries than 2-CFA. The summary-based approach thus enables, via our equivalence result, to measure the precision of $k$-CFA with unbounded $k$, for the class of interprocedural analyses that perform a lossy join.
\end{abstract}

\section{Introduction}

Two dominant approaches to interprocedural static analysis are the summarybased approach and the call-strings approach. Both approaches aim to analyze each procedure precisely by distinguishing calling contexts of a certain kind. But they differ radically in the kind of contexts used: the summary-based (or functional) approach uses input abstract states whereas the call-strings (or $k$-CFA) approach uses sequences of calls that represent call stacks.

Sharir and Pnueli SP81] showed that, in the case of a finite, distributive analysis, the summary-based approach is equivalent to the unbounded call-strings approach (hereafter called $\infty$-CFA). In this case, both these approaches maintain at most one abstract state at each program point under a given context of its containing procedure, applying a join operation to combine different abstract states at each program point into a single state. The distributivity condition ensures that this join is lossless. As a result, both approaches compute the precise meet-over-all-valid-paths (MVP) solution, and are thus equivalent.

Many useful static analyses using the summary-based approach, however, lack distributivity. They too use a join, in order to maintain at most one abstract 
state at each program point under a given context, and thereby scale to large programs (e.g., [FYD $\left.{ }^{+} 08\right]$ ). But in this non-distributive case, the join is lossy, leading such analyses to compute a solution less precise than the MVP solution.

We study the relationship between the summary-based and call-strings approaches, in the presence of a lossy join. Our main result is that these two approaches are equivalent in precision despite their use of radically different ways to distinguish procedure contexts. This result yields both theoretical and practical insights. The theoretical insight includes two new proof techniques. The first is a form of garbage collection on the results computed by the non-distributive summary-based approach. This garbage collection removes entries of procedure summaries that are used during analysis but not in the final analysis results. It provides a natural way for connecting the results of the summary-based approach with those of $\infty$-CFA. The other is a new technique for proving that a fixpoint of a non-monotone function is approximated by a pre-fixpoint of the function. Standard proof techniques do not apply because of non-monotonicity, but such an approximation result is needed in our case because non-distributive summary-based analyses use non-monotone transfer functions.

On the practical side, our equivalence result provides, for the class of nondistributive interprocedural analyses, a feasible approach to determine how precise $k$-CFA can get using arbitrary $k$. This feasible approach is the summary-

based one, which scales much better than $k$-CFA. State-of-the-art algorithms for $k$-CFA do not scale to beyond small values of $k$, as the number of call-string contexts in which they analyze procedures grows exponentially with $k$. As a concrete example, we compare the performance of the summary-based approach to an optimized BDD-based implementation of $k$-CFA for a non-distributive pointer analysis for object-oriented programs. On ten Java programs each of size 400K900K bytecodes from the DaCapo benchmark suite, we find that the $k$-CFA implementation does not scale beyond $k=2$, and even for $k=2$, it computes $4 \mathrm{X}-7 \mathrm{X}$ more contexts per benchmark than the summary-based approach. Furthermore, for three clients of the pointer analysis - downcast safety, call graph reachability, and monomorphic call inference - the summary-based approach proves up to $46 \%$ more client queries per benchmark than 2-CFA, providing an upper bound on the number of queries that is provable by $k$-CFA using arbitrary $k$.

\section{Example}

We illustrate various interprocedural approaches by means of a pointer analysis on the Java program in Figure 1 . All the approaches infer points-to informationaliasing relationships among program variables and heap objects-but differ in their treatment of methods. We illustrate five key aspects of these approaches: (i) 0 -CFA produces imprecise results; (ii) using $k$-CFA with $k>0$ helps to address this imprecision but hurts scalability; (iii) summary-based analysis (hereafter called SBA) causes no loss in precision compared to $k$-CFA; (iv) the lossy join operation in SBA allows analyzing methods in fewer contexts and thereby improves scalability; and (v) SBA can merge multiple $k$-CFA contexts of a method into a single SBA context which also improves scalability. 
analysis based on 1-CFA analyzes a method multiple times, once for each of its call sites. Hence, when it is applied to our example, it differentiates two call sites to add() (i.e., i1 and i2), and analyzes add() twice, once for the call site i1 with the assumption $\left[x \rightarrow\left\{h_{2}\right\}\right]$ on the parameter $x$, and again for the call site i2 with the assumption $\left[x \rightarrow\left\{h_{4}\right\}\right]$. This differentiation enables the analysis to infer that the returned container from foo() contains objects of the type A only, and also that $\mathrm{B}$ : : equals () is not reachable from foo(). In other words, both kinds of imprecision of 0-CFA are eliminated with 1-CFA.

An alternative solution to the imprecision issue is to use SBA. Unlike $k$-CFA, SBA does not distinguish contexts based on sequences of call sites stored in the call stack. Instead, it decides that two calling contexts differ when the abstract states at call sites are different. SBA re-analyzes a method in a calling context only if it has not seen the abstract state $\tau$ of this context before. In Figure 1 the abstract states at call sites i1 and i2 are, respectively, $\left[s_{1} \rightarrow\left\{h_{1}\right\}, a \rightarrow\left\{h_{2}\right\}\right]$ and $\left[s_{2} \rightarrow\left\{h_{3}\right\}, b \rightarrow\left\{h_{4}\right\}\right]$, which become the following input abstract states to add () after the actual parameters are replaced with the formal parameters; $\left[\right.$ this $\left.\rightarrow\left\{h_{1}\right\}, x \rightarrow\left\{h_{2}\right\}\right]$ and $\left[\right.$ this $\left.\rightarrow\left\{h_{3}\right\}, x \rightarrow\left\{h_{4}\right\}\right]$. Since these inputs are different, SBA analyzes method add() separately for the calls from i1 and i2, and reaches the same conclusion about the return value of $f \circ o()$ and call graph reachability as that of 1-CFA described previously. This agreement in analysis results is not an accident. We prove in Section 3 that SBA's results always coincide with those of $\infty$-CFA, a version of $k$-CFA that does not put a bound on the length of call-strings.

An important feature of SBA is that at every control-flow join point in a program, incoming abstract states to this point are combined to a single abstract state via a lossy join operator (if they all originate from the same input abstract state to the current method). This greatly helps the scalability of SBA, because it leads to fewer distinct abstract states at call sites and reduces the number of times that each method should be analyzed. For instance, when SBA analyzes the program in Figure 1 , it encounters two incoming abstract states at the join point $\mathbf{j} 1, \tau_{1}=\left[s \rightarrow\left\{h_{1}\right\}\right]$ from the true branch and $\tau_{2}=\left[s \rightarrow\left\{h_{3}\right\}\right]$ from the false branch. The analysis combines $\tau_{1}$ and $\tau_{2}$ using a lossy join operator, and results in $\tau^{\prime}=\left[s \rightarrow\left\{h_{1}, h_{3}\right\}\right]$. As a result, at the subsequent call site $\mathbf{i 5}$, the analysis has only one input abstract state $\tau^{\prime}$, instead of two (i.e., $\tau_{1}$ and $\tau_{2}$ ), and it analyzes the method taz() only once.

Using a lossy join operator differentiates SBA from the well-known distributive summary-based analysis [RHS95, SP81, which uses a lossless join. If such an analysis were applied to our program, it would collect $\tau_{1}, \tau_{2}$ as the set $\left\{\tau_{1}, \tau_{2}\right\}$ at the join point $\mathbf{j} \mathbf{1}$, and analyze the call to $\operatorname{taz}()$ twice. As a result, the coincidence between the results of SBA and $\infty$-CFA does not follow from what was established previously by Sharir and Pnueli. In fact, proving it requires new proof techniques, as we explain in Section 3.

According to our experiments reported in Section 5 . SBA scales better than $k$ CFA for high $k$ values. This is because SBA usually distinguishes calling contexts of a method less than $k$-CFA, and re-analyzes the method less often than $k$-CFA. 


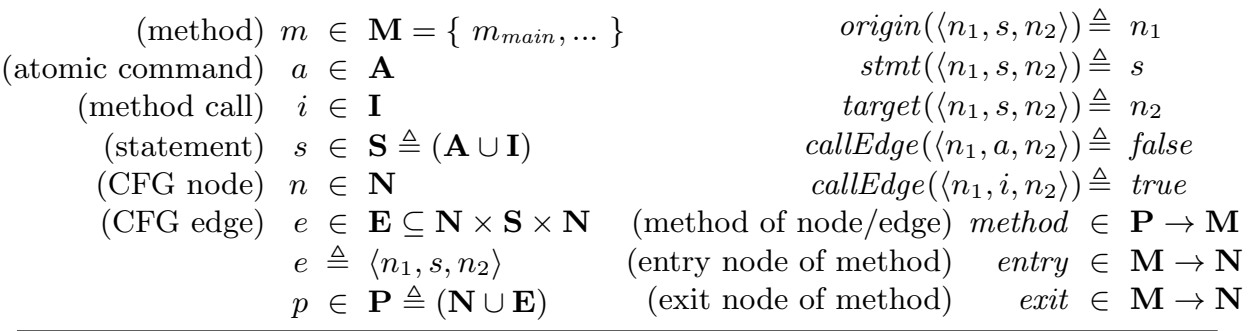

Fig. 2: Notation for interprocedural control flow graphs.

Concretely, a method may be invoked multiple times with call stacks storing different sequences of call sites but with the same abstract state. In this case, $k$-CFA re-analyzes the method for each call sequence in the stack, but SBA analyzes the method only once and reuses the computed summary for all the invocations of this method. In effect, SBA merges multiple $k$-CFA contexts into a single SBA context in this case. This phenomenon can be seen in Figure 1 at the two calls to isEmpty() in i3 and i4. Since these call sites are different, isEmpty () would be analyzed twice by $k$-CFA with $k \geq 1$. However, the abstract state at both of the call sites is the same $\left[s \rightarrow\left\{h_{1}, h_{3}\right\}\right]$. Hence, SBA analyzes the method only once and reuses the computed summary for the second call.

\section{Formal Description and Correspondence Theorem}

This section formalizes an unbounded $k$-CFA and a summary-based interprocedural analysis. The former is an idealization of usual $k$-CFA that does not put a bound on the length of tracked call strings (i.e., sequences of call sites in the call stack), and records analysis results separately for each call string. To emphasize the absence of bound, we call this analysis $\infty$-CFA. The summary-based analysis is a non-distributive variant of the standard summary-based approach for distributive (and disjunctive) analyses [RHS95]. It treats join points approximately using a lossy join operator, unlike the standard approach, and trades precision for performance. The main result of the section is that the summary-based analysis has the same precision as $\infty$-CFA, despite the lossy join.

\subsection{Interprocedural Control Flow Graph}

In our formalism, we assume that programs are specified in terms of interprocedural control flow graphs $\mathcal{G}=(\mathbf{M}, \mathbf{A}, \mathbf{I}, \mathbf{N}, \mathbf{E}$, method, entry, exit $)$ in Figure 2 . Set $\mathbf{M}$ consists of method names in a program, and $\mathbf{A}$ and $\mathbf{I}$ specify available atomic commands and method call instructions. Sets $\mathbf{N}$ and $\mathbf{E}$ determine nodes and intraprocedural edges of a control flow graph. Each node in this graph belongs to a method given by the function method. The functions entry and exit decide the entry and exit nodes of each method. The figure also shows defined entities-origin, stmt, target, and callEdge, which can be used to obtain components of an edge and to decide the type of the edge. We assume all the five sets in a control flow graph are finite. 


$$
\begin{aligned}
& \text { (abstract state) } \quad \tau \in \boldsymbol{\Gamma}=\left\{\tau_{\text {init }}, \ldots\right\} \\
& \text { (lattice operations) } \bigsqcup, \Pi \in \mathcal{P}(\boldsymbol{\Gamma}) \rightarrow \boldsymbol{\Gamma} \perp, \top \in \boldsymbol{\Gamma} \sqsubseteq \subseteq \boldsymbol{\Gamma} \times \boldsymbol{\Gamma} \\
& \text { (transfer functions) } \quad \llbracket a \rrbracket \in \Gamma \rightarrow \Gamma \\
& \text { (targets of call) calls }(s, \tau) \in \mathcal{P}(\mathbf{M}) \\
& \text { (call string) } \quad \pi \in \mathbf{\Pi} \triangleq \bigcup_{n \geq 0}(\mathbf{M} \cup \mathbf{E})^{n} \\
& \left(\infty \text {-CFA annotation) } \quad \kappa \in \mathbf{A}_{\mathrm{cfa}}=(\mathbf{P} \times \mathbf{\Pi}) \rightarrow \boldsymbol{\Gamma}\right. \\
& \text { (SBA annotation) } \quad \sigma \in \mathbf{A}_{\text {sba }}=(\mathbf{P} \times \boldsymbol{\Gamma}) \rightarrow \boldsymbol{\Gamma}
\end{aligned}
$$

Fig. 3: Analysis domains and transfer functions.

$$
\begin{aligned}
& F_{\mathrm{cfa}}(\kappa)(n, \pi)= \begin{cases}\bigsqcup\{\kappa(e, \pi) \mid n=\operatorname{target}(e)\} & \text { if } \nexists m: n=\text { entry }(m) \\
\bigsqcup\left\{\tau \mid \exists e, \pi_{1}: \text { callEdge }(e) \wedge \pi=m \oplus e \oplus \pi_{1}\right. & \text { if } n=\operatorname{entry}(m) \\
\left.\wedge \tau=\kappa\left(\text { origin }(e), \pi_{1}\right) \wedge m \in \operatorname{calls}(\operatorname{stmt}(e), \tau)\right\} & \text { if } \neg \text { callEdge }(e)\end{cases} \\
& F_{\mathrm{cfa}}(\kappa)(e, \pi)= \begin{cases}\llbracket \operatorname{stmt}(e) \rrbracket(\kappa(\text { origin }(e), \pi)) & \text { if callEdge }(e) \\
\bigsqcup\left\{\tau \mid \exists \tau_{1}, m: \tau_{1}=\kappa(\operatorname{origin}(e), \pi)\right. & \\
\left.\wedge m \in \operatorname{calls}\left(\operatorname{stmt}(e), \tau_{1}\right) \wedge \tau=\kappa(\operatorname{exit}(m), m \oplus e \oplus \pi)\right\}\end{cases}
\end{aligned}
$$

Fig. 4: Transfer function $F_{\text {cfa }}$ on $\infty$-CFA annotations.

Our control flow graphs are required to satisfy well-formedness conditions. First, $m_{\text {main }} \in \mathbf{M}$. Second, for all $m \in \mathbf{M}$ and $e \in \mathbf{E}$,

$$
\begin{aligned}
& \text { entry }(m) \neq \operatorname{exit}(m) \wedge(\operatorname{method} \circ \text { entry })(m)=(\operatorname{method} \circ \text { exit })(m)=m \wedge \\
& (\operatorname{method} \circ \text { target })(e)=(\operatorname{method} \circ \operatorname{origin})(e)=\operatorname{method}(e) .
\end{aligned}
$$

The first conjunct means that the entry node and the exit node of a method are different, the second says that entry and exit pick nodes belonging to their argument method, and the last conjunct states that an edge and its source and target nodes are in the same method.

\subsection{Formal Description of Analyses}

Both $\infty$-CFA and the summary-based analysis assume $\left(\boldsymbol{\Gamma}, \tau_{\text {init }}, \llbracket-\rrbracket\right.$, calls $)$ in Figure 3. which are needed for performing an intraprocedural analysis as well as processing dynamically dispatched method calls. Component $\boldsymbol{\Gamma}$ is a finite complete lattice, and consists of abstract states used by the analysis. The next $\tau_{\text {init }} \in \boldsymbol{\Gamma}$ is an initial abstract state to the root method $m_{\text {main }}$, and $\llbracket a \rrbracket$ represents abstract transfer functions for atomic commands $a$. The final component calls takes a pair $(s, \tau)$, and conservatively estimates target methods of a call $s$ in (concrete) states described by $\tau$, if $s$ is a method call. Otherwise, it returns the empty set.

We require that the components of the analysis satisfy the following properties: (i) $\tau_{\text {init }} \neq \perp$; (ii) calls $\left(s,{ }_{-}\right)$and $\llbracket a \rrbracket$ are monotone with respect to the order in $\Gamma$ or the subset order ${ }^{\top}$, (iii) calls $(s, \perp)=\emptyset$ and $\llbracket a \rrbracket(\perp)=\perp$; (iv) for all $s$ and $\tau, m_{\text {main }} \notin$ calls $(s, \tau)$, and if $s$ is not a method call, calls $(s, \tau)=\emptyset$.

$\infty$-CFA Analysis. The $\infty$-CFA analysis is an interprocedural analysis that uses call strings of arbitrary length as calling contexts and analyzes a method

\footnotetext{
$\overline{{ }^{1} \text { This means }} \forall \tau, \tau^{\prime} \in \Gamma: \tau \sqsubseteq \tau^{\prime} \Longrightarrow\left(\operatorname{calls}(s, \tau) \subseteq \operatorname{calls}\left(s, \tau^{\prime}\right) \wedge \llbracket a \rrbracket(\tau) \sqsubseteq \llbracket a \rrbracket\left(\tau^{\prime}\right)\right)$.
} 


$$
\begin{aligned}
& F_{\text {sba }}(\sigma)(n, \tau)= \begin{cases}\sqcup\{\sigma(e, \tau) \mid n=\operatorname{target}(e)\} & \text { if } \nexists m: n=\operatorname{entry}(m) \\
\sqcup\left\{\tau \mid \exists e, \tau_{1}: \operatorname{callEdge}(e) \wedge \tau=\sigma\left(\operatorname{origin}(e), \tau_{1}\right)\right. & \text { if } n=\operatorname{entry}(m) \\
\wedge m \in \operatorname{calls}(\operatorname{stmt}(e), \tau)\} & \end{cases} \\
& F_{\text {sba }}(\sigma)(e, \tau)=\left\{\begin{array}{cl}
\llbracket \operatorname{stmt}(e) \rrbracket(\sigma(\operatorname{origin}(e), \tau)) & \text { if } \neg \text { callEdge }(e) \\
\sqcup\left\{\tau^{\prime} \mid \exists \tau_{1}, m: \tau_{1}=\sigma(\operatorname{origin}(e), \tau)\right. & \text { if callEdge }(e) \\
\left.\wedge m \in \operatorname{calls}\left(\operatorname{stmt}(e), \tau_{1}\right) \wedge \tau^{\prime}=\sigma\left(\operatorname{exit}(m), \tau_{1}\right)\right\} &
\end{array}\right.
\end{aligned}
$$

Fig. 5: Transfer function $F_{\text {sba }}$ on SBA annotations.

separately for each call string. If a reader is familiar with $k$-CFA, we suggest to view $\infty$-CFA as the limit of $k$-CFA with $k$ tending towards $\infty$. Indeed, $\infty$-CFA computes a result that is as precise as any $k$-CFA analysis.

The $\infty$-CFA works by repeatedly updating a map $\kappa \in \mathbf{A}_{\mathrm{cfa}}=(\mathbf{P} \times \boldsymbol{\Pi}) \rightarrow \boldsymbol{\Gamma}$, called $\infty$-CFA annotation. The first argument $p$ to $\kappa$ is a program node or an edge, and the second $\pi$ a call string defined in Figure 3 , which is a finite sequence of method names and edges. A typical call string is $m_{2} \oplus e_{2} \oplus m_{1} \oplus e_{1} \oplus m_{\text {main }}$. It represents a chain of calls $m_{\text {main }} \rightarrow m_{1} \rightarrow m_{2}$, where $m_{1}$ is called by the edge $e_{1}$ and $m_{2}$ by $e_{2}$. The function $\kappa$ maps such $p$ and $\pi$ to an abstract state $\tau$, the current estimation of concrete states reaching $p$ with $\pi$ on the call stack.

We order $\infty$-CFA annotations pointwise: $\kappa \sqsubseteq \kappa^{\prime} \Longleftrightarrow \forall p, \pi: \kappa(p, \pi) \sqsubseteq \kappa^{\prime}(p, \pi)$. This order makes the set of $\infty$-CFA annotations a complete lattice. The $\infty$-CFA analysis computes a fixpoint on $\infty$-CFA annotations:

$$
\kappa_{\text {cfa }}=\text { leastFix } \lambda \kappa .\left(\kappa_{I} \sqcup F_{\text {cfa }}(\kappa)\right) .
$$

Here $\kappa_{I}$ is the initial $\infty$-CFA annotation, and models our assumption that a given program starts at $m_{\text {main }}$ in a state satisfying $\tau_{\text {init }}$ :

$$
\kappa_{I}(p, \pi)=\text { if }\left((p, \pi)=\left(\operatorname{entry}\left(m_{\text {main }}\right), m_{\text {main }}\right)\right) \text { then } \tau_{\text {init }} \text { else } \perp .
$$

Function $F_{\text {cfa }}$ is the so called transfer function, and overapproximates one-step execution of atomic commands and method calls in a given program. Figure 4 gives the definition of $F_{\text {cfa }}$. Although this definition looks complicated, it comes from a simple principle: $F_{\text {cfa }}$ updates its input $\kappa$ simply by propagating abstract states in $\kappa$ to appropriate next nodes or edges, while occasionally pushing or popping call sites and invoked methods in the tracked call string.

We make two final remarks on $\infty$-CFA. First, $F_{\text {cfa }}$ is monotone with respect to our order on $\infty$-CFA annotations. This ensures that the least fixpoint in (1) exists. Although the monotonicity is an expected property, we emphasize it here because the transfer function of our next interprocedural analysis SBA is not monotone with respect to a natural order on analysis results. Second, the domain of $\infty$-CFA annotations is infinite, so a finite number of iterations might be insufficient for reaching the least fixpoint in (1). We are not concerned with this potential non-computability, because we use $\infty$-CFA only as a device for comparing the precision of SBA in the next subsection with that of $k$-CFA.

Summary-Based Analysis. The summary-based analysis SBA is another approach to analyze methods context-sensitively. Just like $\infty$-CFA, it keeps sep- 
arate analysis results for different calling contexts, but differs from $\infty$-CFA in that it uses input abstract states to methods as contexts, instead of call strings.

The main data structures of SBA are SBA annotations $\sigma$ :

$$
\sigma \in \mathbf{A}_{\mathrm{sba}}=(\mathbf{P} \times \boldsymbol{\Gamma}) \rightarrow \boldsymbol{\Gamma} .
$$

An SBA annotation $\sigma$ specifies an abstract state $\sigma(p, \tau)$ at each program point $p$ for each calling context $\tau$. Recall that a calling context here is just an initial abstract state to the current method. SBA annotations are ordered pointwise: $\sigma \sqsubseteq \sigma^{\prime} \Longleftrightarrow \forall p, \tau: \sigma(p, \tau) \sqsubseteq \sigma^{\prime}(p, \tau)$. With this order, the set of SBA annotations forms a complete lattice. Further, it is finite as $\mathbf{P}$ and $\boldsymbol{\Gamma}$ are finite.

The summary-based analysis is an iterative algorithm for computing a fixpoint of some function on SBA annotations. It starts with setting the current SBA annotation to $\sigma_{I}$ below:

$$
\sigma_{I}(p, \tau)=\text { if }\left((p, \tau)=\left(\operatorname{entry}\left(m_{\text {main }}\right), \tau_{\text {init }}\right)\right) \text { then } \tau_{\text {init }} \text { else } \perp,
$$

which says that only the entry node of $m_{\text {main }}$ has the abstract state $\tau_{\text {init }}$ under the context $\tau_{\text {init }}$. Then, it repeatedly updates the current SBA annotation using the transfer function $F_{\text {sba }}$ in Figure 5 The function propagates abstract states at all program nodes and edges along interprocedural control-flow edges. In doing so, it approximates one-step execution of every atomic command and method call in a given program. The summary-based analysis does the following fixpoint computation and calculates $\sigma_{\text {sba }}$ :

$$
\sigma_{\text {sba }}=\mathrm{fix}^{\sigma_{I}}\left(\lambda \sigma . \sigma \sqcup F_{\text {sba }}(\sigma)\right) .
$$

Let $G=\left(\lambda \sigma . \sigma \sqcup F_{\text {sba }}(\sigma)\right)$. Here (fix $\left.{ }^{\sigma_{I}} G\right)$ generates the sequence $G^{0}\left(\sigma_{I}\right), G^{1}\left(\sigma_{I}\right)$, $G^{2}\left(\sigma_{I}\right), \ldots$, until it reaches a fixpoint $G^{n}\left(\sigma_{I}\right)$ such that $G^{n}\left(\sigma_{I}\right)=G^{n+1}\left(\sigma_{I}\right)$. This fixpoint $G^{n}\left(\sigma_{I}\right)$ becomes the result $\sigma_{\text {sba }}$ of fix ${ }^{\sigma_{I}} G$.

Note that fix always reaches a fixpoint in (2). The generated sequence is always increasing because $\sigma \sqsubseteq G(\sigma)$ for every $\sigma$. Since the domain of SBA annotations is finite, this increasing sequence should reach a fixpoint. One might wonder why SBA does not use the standard least fixpoint. The reason is that our transfer function $F_{\text {sba }}$ is not monotone, so the standard theory for least fixpoints does not apply. This is in contrast to $\infty$-CFA that has the monotone transfer function. Non-monotone transfer functions commonly feature in program analyses for numerical properties that use widening operators [Min06, CC92, and the results of these analyses are computed similarly to what we described above (modulo the additional use of a widening operator).

\subsection{Correspondence Theorem}

The main result of this section is the Correspondence Theorem, which says that $\infty$-CFA and SBA have the same precision.

Recall that the results of SBA and $\infty$-CFA are functions of different types: the domain of $\sigma_{\text {sba }}$ is $\mathbf{P} \times \boldsymbol{\Gamma}$, whereas that of $\kappa_{\text {cfa }}$ is $\mathbf{P} \times \boldsymbol{\Pi}$. Hence, to connect the results of both analyses, we need a way to relate functions of the first kind with those of the second. For this purpose, we use a particular kind of functions: 
Definition $1 A$ translation function $\eta$ is a map of type $\mathbf{M} \times \boldsymbol{\Pi} \rightarrow \boldsymbol{\Gamma}$.

Intuitively, $\eta(m, \pi)=\tau$ expresses that although a call string $\pi$ and an abstract state $\tau$ are different types of calling contexts, we will treat them the same when they are used as contexts for method $m$.

One important property of a translation function $\eta$ is that it induces maps between SBA and $\infty$-CFA annotations:

$$
\begin{array}{ll}
L(\eta,-): \mathbf{A}_{\mathrm{sba}} \rightarrow \mathbf{A}_{\mathrm{cfa}} & L(\eta, \sigma)=\lambda(p, \pi) . \sigma(p, \eta(\operatorname{method}(p), \pi)), \\
R(\eta,-): \mathbf{A}_{\mathrm{cfa}} \rightarrow \mathbf{A}_{\mathrm{sba}} & R(\eta, \kappa)=\lambda(p, \tau) . \sqcap\{\kappa(p, \pi) \mid \tau \sqsubseteq \eta(\operatorname{method}(p), \pi)\} .
\end{array}
$$

Both $L$ and $R$ use $\eta$ to convert calling contexts of one type to those of the other. The conversion in $L(\eta, \sigma)$ is as we expect; it calls $\eta$ to change an input call string $\pi$ to an input abstract state $\eta(\operatorname{method}(p), \pi)$, which is then fed to the given SBA annotation $\sigma$. On the other hand, the conversion in $R(\eta, \kappa)$ is unusual, but follows the same principle of using $\eta$ for translating contexts. Conceptually, it changes an input abstract state $\tau$ to a set of call strings $\pi$ that would be translated to an overapproximation of $\tau$ by $\eta$ (i.e., $\tau \sqsubseteq \eta(\operatorname{method}(p), \pi))$, looks up the values of $\kappa$ at these call strings, and combine the looked-up values by the meet operation. The following lemma relates $L(\eta,-)$ and $R(\eta,-)$ :

Lemma 1. For all $\sigma$ and $\kappa$, if $\sigma \sqsubseteq R(\eta, \kappa)$, then $L(\eta, \sigma) \sqsubseteq \kappa$.

The definition of a translation function does not impose any condition, and permits multiple possibilities. Hence, a natural question is: what is a good translation function $\eta$ that would help us to relate the results of the SBA analysis with those of the $\infty$-CFA analysis? The following lemma suggests one such candidate $\eta_{\text {sba }}$, which is constructed from the results $\sigma_{\text {sba }}$ of the SBA analysis:

Lemma 2. There exists a unique translation function $\eta: \mathbf{M} \times \mathbf{\Pi} \rightarrow \boldsymbol{\Gamma}$ such that for all $m \in \mathbf{M}, e \in \mathbf{E}$ and $\pi \in \mathbf{\Pi}$,

$$
\begin{aligned}
& \eta\left(m_{\text {main }}, m_{\text {main }}\right)=\tau_{\text {init }}, \\
& \begin{array}{c}
\eta(m, m \oplus e \oplus \pi)=\text { if }\left(m \in \operatorname{calls}\left(\operatorname{stmt}(e), \sigma_{\text {sba }}(\operatorname{origin}(e), \eta(\operatorname{method}(e), \pi))\right)\right. \\
\quad \wedge \text { callEdge }(e)) \text { then } \sigma_{\text {sba }}(\operatorname{origin}(e), \eta(\operatorname{method}(e), \pi)) \text { else } \perp, \\
\eta(m, \pi)=\perp \quad(\text { for all the other cases }) .
\end{array}
\end{aligned}
$$

We denote this translation with $\eta_{\text {sba }}$.

Intuitively, for each call string $\pi$, the translation $\eta_{\text {sba }}$ in the lemma follows the chain of calls in $\pi$ while tracking corresponding abstract input states stored in $\sigma$. When this chasing is over, it finds an input abstract state $\tau$ corresponding to the given $\pi$. For instance, given a method $m_{2}$ and a call string $m_{2} \oplus e_{2} \oplus m_{1} \oplus e_{1} \oplus$ $m_{\text {main }}$, if all the side conditions in the lemma are met, $\eta_{\text {sba }}$ returns abstract state $\sigma_{\text {sba }}\left(\operatorname{origin}\left(e_{2}\right), \sigma_{\text {sba }}\left(\operatorname{origin}\left(e_{1}\right), \tau_{\text {init }}\right)\right)$. This corresponds to the input abstract state to method $m_{2}$ that arises after method calls first at $e_{1}$ and then $e_{2}$.

Another good intuition is to view $\eta_{\mathrm{sba}}$ as a garbage collector. Specifically, for each method $m$, the set

$$
\Gamma_{m}=\left\{\eta_{\text {sba }}(m, \pi) \mid \pi \in \boldsymbol{\Pi}\right\} .
$$


identifies input abstract states for $m$ that contribute to the analysis result $\sigma_{\text {sba }}$ along some call chain from $m_{\text {main }}$ to $m$; every other input abstract state $\tau$ for $m$ is garbage even if it was used during the fixpoint computation of $\sigma_{\text {sba }}$ and so $\sigma_{\text {sba }}(\operatorname{entry}(m), \tau) \neq \perp$.

Our Correspondence Theorem says that the SBA analysis and the $\infty$-CFA analysis compute the same result modulo the translation via $L\left(\eta_{\text {sba }},-\right)$.

Theorem 2 (Correspondence). $L\left(\eta_{\mathrm{sba}}, \sigma_{\mathrm{sba}}\right)=\kappa_{\mathrm{cfa}}$.

One important consequence of this theorem is that both analyses have the same estimation about reachable concrete states at each program point, if we garbagecollect the SBA's result using $\eta_{\text {sba }}$ :

Corollary 1. For all $p \in \mathbf{P}$ and $m \in \mathbf{M}$, if $\operatorname{method}(p)=m$, then

$$
\left\{\kappa_{\text {cfa }}(p, \pi) \mid \pi \in \boldsymbol{\Pi}\right\}=\left\{\sigma_{\text {sba }}\left(p, \tau^{\prime}\right) \mid \tau^{\prime} \in \Gamma_{m}\right\} \text {, where } \Gamma_{m} \text { is defined by (3). }
$$

Overview of Proof of the Correspondence Theorem. Proving the Correspondence Theorem is surprisingly tricky. A simple proof strategy is to show that the relationship in the theorem is maintained by each step of the fixpoint computations of $\infty$-CFA and SBA, but this strategy does not work. Since $\infty$-CFA and SBA treat the effects of method calls (i.e., call edges) very differently, the relationship in the theorem is not maintained during fixpoint computations. Further difficulties arise because the SBA analysis uses a non-monotone transfer function $F_{\text {sba }}$ and does not necessarily compute the least fixpoint of $\lambda \sigma . \sigma_{I} \sqcup F_{\text {sba }}(\sigma)$ - these render standard techniques for reasoning about fixpoints no longer applicable.

In this subsection, we outline our proof of the Correspondence Theorem, and point out proof techniques that we developed to overcome difficulties mentioned above. The full proof is included in the Appendix.

Let $G_{\mathrm{cfa}}=\lambda \kappa . \kappa_{I} \sqcup F_{\kappa}(\kappa)$ and $G_{\text {sba }}=\lambda \sigma . \sigma_{I} \sqcup F_{\text {sba }}(\sigma)$. Recall that the $\infty$-CFA analysis computes the least fixpoint of $G_{\mathrm{cfa}}$ while the SBA analysis computes some pre-fixpoint of $G_{\text {sba }}$ (i.e., $\left.G_{\text {sba }}\left(\sigma_{\text {sba }}\right) \sqsubseteq \sigma_{\text {sba }}\right)$ via an iterative process. Our proof consists of the following four main steps.

1. First, we prove that $G_{\mathrm{cfa}}\left(L\left(\eta_{\mathrm{sba}}, \sigma_{\mathrm{sba}}\right)\right) \sqsubseteq L\left(\eta_{\mathrm{sba}}, \sigma_{\mathrm{sba}}\right)$. That is, $L\left(\eta_{\mathrm{sba}}, \sigma_{\mathrm{sba}}\right)$ is a pre-fixpoint of $G_{\mathrm{cfa}}$. This implies

$$
\kappa_{\mathrm{cfa}} \sqsubseteq L\left(\eta_{\mathrm{sba}}, \sigma_{\mathrm{sba}}\right),
$$

a half of the conclusion in the Correspondence Theorem. To see this implication, note that the function $G_{\mathrm{cfa}}$ is monotone and works on a complete lattice, and the analysis computes the least fixpoint $\kappa_{\text {cfa }}$ of $G_{\text {cfa }}$. According to the standard result, the least fixpoint is also the least pre-fixpoint, so $\kappa_{\text {cfa }}$ is less than or equal to another pre-fixpoint $L\left(\eta_{\text {sba }}, \sigma_{\text {sba }}\right)$.

2 . We next construct another translation, denoted $\eta_{\mathrm{cfa}}$, this time from the result of the $\infty$-CFA analysis: $\eta_{\text {cfa }}=\lambda(m, \pi) . \kappa_{\text {cfa }}(\operatorname{entry}(m), \pi)$. Then, we show

$$
\sigma_{\mathrm{sba}} \sqsubseteq R\left(\eta_{\mathrm{cfa}}, \kappa_{\mathrm{cfa}}\right) .
$$

The proof of this inequality uses our new technique for verifying that an SBA annotation overapproximates $\sigma_{\text {sba }}$, a pre-fixpoint of a non-monotone function $G_{\text {sba. }}$. We will explain this technique at the end of this subsection. 
3. Third, we apply Lemma 1 to the inequality in (5), combine the result of this application with (4), and derive

$$
L\left(\eta_{\text {cfa }}, \sigma_{\text {sba }}\right) \sqsubseteq \kappa_{\text {cfa }} \sqsubseteq L\left(\eta_{\text {sba }}, \sigma_{\text {sba }}\right) .
$$

4. Finally, using the relationship between $\sigma_{\text {sba }}$ and $\kappa_{\text {cfa }}$ in $(6)$, we show that $\eta_{\text {cfa }}=\eta_{\text {sba }}$. Note that conjoined with the same relationship again, this equality entails $L\left(\eta_{\text {sba }}, \sigma_{\text {sba }}\right)=\kappa_{\text {cfa }}$, the claim of the Correspondence theorem.

Before finishing, let us explain a proof technique used in the second step. An SBA annotation $\sigma$ is monotone if $\forall p, \tau, \tau^{\prime}: \tau \sqsubseteq \tau^{\prime} \Longrightarrow \sigma(p, \tau) \sqsubseteq \sigma\left(p, \tau^{\prime}\right)$. Our proof technique is summarised in the following lemma:

Lemma 3. For all $S B A$ annotations $\sigma$, if $\sigma$ is monotone, $G_{\text {sba }}(\sigma) \sqsubseteq \sigma$ and

$$
\forall m: \tau \sqsubseteq \sigma(\operatorname{entry}(m), \tau),
$$

then $\sigma_{\text {sba }} \sqsubseteq \sigma$.

We remind the reader that if $G_{\text {sba }}$ is a monotone function and $\sigma_{\text {sba }}$ is its least fixpoint, we do not need the monotonicity of $\sigma$ and the condition in (7) in the lemma. In this case, $\sigma_{\text {sba }} \sqsubseteq \sigma$ even without these conditions. This lemma extends this result to the non-monotone case, and identifies additional conditions.

The conclusion of the second step in our overview above is obtained using Lemma 3 . In that step, we prove that (1) $R\left(\eta_{\mathrm{cfa}}, \kappa_{\mathrm{cfa}}\right)$ is monotone; (2) it is a pre-fixpoint of $G_{\text {sba }}$; (3) it satisfies the condition in (7). Hence, Lemma3 3 applies, and gives $\sigma_{\text {sba }} \sqsubseteq R\left(\eta_{\text {cfa }}, \kappa_{\text {cfa }}\right)$.

A final comment is that when the abstract domain of a static analysis is infinite, if it is a complete lattice, we can still define SBA similar to our current definition. The only change is that the result of SBA, $\sigma_{\mathrm{sba}}$, is now defined in terms of the limit of a potentially infinite chain (generated by the application of $G_{\text {sba }}$ and the least-upper-bound operator for elements at limit ordinals), instead of a finite chain. This new SBA is not necessarily computable, but we can still ask whether its result coincides with that of $\infty$-CFA. We believe that the answer is yes: most parts of our proof seem to remain valid for this new SBA, while the remaining parts (notably the proof of Lemma 3) can be modified relatively easily to accommodate this new SBA. This new Coincidence theorem, however, is limited; it does not say anything about analyses with widening.

\section{Application to Pointer Analysis}

We now show how to apply the summary-based approach to a pointer analysis for object-oriented programs, which also computes the program's call graph.

The input to the analysis is a program in the form of an interprocedural control flow graph (defined in Section 3.1). Figure 6 shows the kinds of statements it considers: atomic commands that create, read, and write pointer locations, via local variables $v$, global variables (i.e., static fields) $g$, and object fields (i.e., instance fields) $f$. We label each allocation site with a unique label $h$. We elide 


\begin{tabular}{|c|c|c|c|c|c|c|c|c|c|}
\hline & \multirow[t]{2}{*}{ brief description } & \multicolumn{2}{|c|}{ classes } & \multicolumn{2}{|c|}{ methods } & \multicolumn{2}{|c|}{ bytecode $(\mathrm{KB})$} & \multicolumn{2}{|c|}{ KLOC } \\
\hline & & app & total & app & total & app & total & app & total \\
\hline antlr & parser/translator generator & 109 & 1,091 & 873 & 7,220 & 81 & 467 & 26 & 224 \\
\hline avrora & microcontroller simulator/analyzer & 78 & 1,062 & 523 & 6,905 & 35 & 423 & 16 & 214 \\
\hline bloat & bytecode optimization/analysis tool & 277 & 1,269 & 2,651 & 9,133 & 195 & 586 & 59 & 258 \\
\hline chart & graph plotting tool and pdf renderer & 181 & 1,756 & 1,461 & 11,450 & 101 & 778 & 53 & 366 \\
\hline hsqldb & SQL relational database engine & 189 & 1,341 & 2,441 & 10,223 & 190 & 670 & 96 & 322 \\
\hline luindex & text indexing tool & 193 & 1,175 & 1,316 & 7,741 & 99 & 487 & 38 & 237 \\
\hline lusearch & text search tool & 173 & 1,157 & 1,119 & 7,601 & 77 & 477 & 33 & 231 \\
\hline pmd & Java source code analyzer & 348 & 1,357 & 2,590 & 9,105 & 186 & 578 & 46 & 247 \\
\hline sunflow & photo-realistic rendering system & 165 & 1,894 & 1,328 & 13,356 & 117 & 934 & 25 & 419 \\
\hline xalan & XSLT processor to transform XML & 42 & 1,036 & 372 & 6,772 & 28 & 417 & 9 & 208 \\
\hline
\end{tabular}

Table 1: Program statistics by flow and context insensitive call graph analysis $(0 \mathrm{CFA} I)$.

to sets of locals. We explain the most interesting ones. Equation 12 states that cast statement $v^{\prime}=(t) v$ sets the points-to set of local $v^{\prime}$ after the statement to those allocation sites in the points-to set of $v$ before the statement that are subtypes of $t$. Equations (13) and (14) are transfer functions for statements that read globals and fields. Since ptsV tracks points-to information only for locals, we use separate data ptsG and ptsF to track points-to information for globals and fields, respectively. These data are updated by transfer functions for statements that write globals and fields, shown in Equations (16) and (17). Since the transfer functions both read and write data ptsG and ptsF, the algorithm for our combined points-to and call graph analysis has an outer loop that calls the SBA algorithm from Section 3 until ptsG and ptsF reach a fixpoint, starting with empty data for them in the initial iteration, $\lambda g . \emptyset$ and $\lambda(h, f) . \emptyset$. This outer loop implements a form of the reduced product CC79] of our flow-sensitive points-to analysis for locals and the flow-insensitive analysis for globals and fields ${ }^{2}$ It is easy to see that the resulting algorithm terminates as $\boldsymbol{\Gamma}$ is finite.

Finally, in addition to points-to information, our analysis produces a context sensitive call graph, denoted by a set cg containing each tuple $\left(\tau_{1}, e, \tau_{2}, m\right)$ such that the call at control-flow edge $e$ in context $\tau_{1}$ of its containing method may call the target method $m$ in context $\tau_{2}$. It is straightforward to compute this information by instrumenting the SBA algorithm to add tuple $\left(\tau_{1}, e, \tau_{2}, m\right)$ to $\mathrm{cg}$ whenever it visits a call site $e$ in context $\tau_{1}$ and computes a target method $m$ and a target context $\tau_{2}$.

\section{Empirical Evaluation}

We evaluated various interprocedural approaches on our pointer analysis using ten Java programs from the DaCapo benchmark suite (http://dacapobench.org), shown in Table 1. All experiments were done using Oracle HotSpot JVM 1.6.0 on a Linux machine with 32GB RAM and AMD Opteron 3.0GHz processor. We also measured the precision of these approaches on three different clients of the pointer analysis. We implemented all our approaches and clients using the Chord

\footnotetext{
${ }^{2}$ We used flow-insensitive analysis for globals and fields to ensure soundness under concurrency - many programs in our experiments are concurrent.
} 


\begin{tabular}{l|c|c|c}
\hline & kind of implementation & context sensitivity degree $(k)$ & flow sensitivity for locals? \\
\hline SBA $_{S}$ & RHS & $\infty$ & yes \\
0CFA $_{S}$ & RHS & 0 & yes \\
kCFA $_{I}$ & BDD & $0,1,2$ & no \\
\hline
\end{tabular}

Table 2: Interprocedural approaches evaluated in our experiments.

program analysis platform for Java bytecode (http://jchord.googlecode.com). We next describe the various approaches and clients.

Interprocedural Approaches. The approaches we evaluated are shown in Table 2. They differ in three aspects: (i) the kind of implementation (tabulation algorithm from RHS95] called RHS for short vs. BDD); (ii) the degree of call-strings context sensitivity (i.e., the value of $k$ ); and (iii) flow sensitive vs. flow insensitive tracking of points-to information for locals. The approach in Section 4 is the most precise one, $\mathrm{SBA}_{S}$. It is a non-distributive summary-based approach that yields unbounded $k$-CFA context sensitivity, tracks points-to information of locals flow sensitively, and does heap updates context sensitively. It is implemented using RHS. Doing context sensitive heap updates entails SBA $_{S}$ calling the tabulation algorithm repeatedly in an outer loop that iterates until points-to information for globals and fields reaches a fixpoint (each iteration of this outer loop itself executes an inner loop - an invocation of the tabulation algorithm - that iterates until points-to information for locals reaches a fixpoint). We confirmed that the non-distributive aspect (i.e., the lossy join) is critical to the performance of our RHS implementation: it ran out of memory on all our benchmarks without lossy join. In fact, the lossy join even obviated the need for other optimizations in our RHS implementation, barring only the use of bitsets to represent points-to sets.

It is easy to derive the remaining approaches in Table 2 from SBA $_{S}$. OCFA is the context insensitive version of $\mathrm{SBA}_{S}$. It also uses the RHS implementation and leverages the flow sensitive tracking of points-to information of locals in the tabulation algorithm. We could not scale the RHS implementation to simulate $k$-CFA for $k>0$. Hence, our evaluation includes a non-RHS implementation: an optimized BDD-based one that tracks points-to information of locals flow insensitively but allows us to do bounded context sensitive $k$-CFA for $k>0$. Even this optimized implementation, however, ran out of memory on all our benchmarks beyond $k=2$. Nevertheless, using it up to $k=2$ enables us to gauge the precision and performance of a state-of-the-art bounded $k$-CFA approach.

To summarize, the relative precision of the approaches we evaluate is: $\mathrm{SBA}_{S} \preceq$

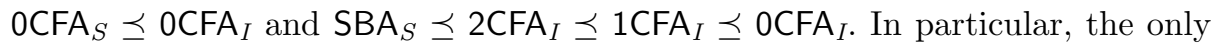
incomparable pairs are $\left(0\right.$ CFA $_{S}, 1$ CFA $)$ and $\left(0 \mathrm{CFA} A_{S}, 2 \mathrm{CFA}_{I}\right)$.

Pointer Analysis Clients. We built three clients that use the result of our pointer analysis: downcast safety, call graph reachability, and monomorphic call site inference. The result used by these clients is the context sensitive call graph, $\mathrm{cg} \subseteq(\mathbf{C} \times \mathbf{E} \times \mathbf{C} \times \mathbf{M})$, and context sensitive points-to sets of locals at each program point, pts $\in(\mathbf{N} \times \mathbf{C}) \rightarrow \boldsymbol{\Gamma}$, where contexts $c \in \mathbf{C}$ are abstract environments (in domain $\boldsymbol{\Gamma}$ ) for the $\mathrm{SBA}_{*}$ approaches and bounded call strings (in 
domain $\boldsymbol{\Pi})$ for the $\mathrm{CFA}_{*}$ approaches. The above result signatures are the most general, for instance, a context insensitive approach like 0CFA ${ }_{I}$ may use a degenerate $\mathbf{C}$ containing a single context, and a flow insensitive approach like $\mathrm{kCFA}_{I}$ may ignore program point $n$ in $\operatorname{pts}(n, c)$, giving the same points-to information at all program points for a local variable of a method under context $c$. We next formally describe our three clients using the above results.

Downcast Safety. This client statically checks the safety of downcasts. A safe downcast is one that cannot fail because the object to which it is applied is guaranteed to be a subtype of the target type. Thus, safe downcasts obviate the need for run-time cast checking. We define this client in terms of the downcast predicate: downcast $(e) \Longleftrightarrow \exists c:\{\operatorname{type}(h) \mid h \in \operatorname{pts}(n, c)(v)\} \nsubseteq \operatorname{sub}(t)$, where the command at control-flow edge $e$ with $\operatorname{origin}(e)=n$ is a cast statement $v^{\prime}=(t) v$. The predicate checks if the type of some allocation site in the pointsto set of $v$ is not a subtype of the target type $t$. Each query to this client is a cast statement at $e$ in the program. It is proven by an analysis if downcast $(e)$ evaluates to false using points-to information pts computed by the analysis.

Call Graph Reachability. This client determines pairwise reachability between every pair of methods. The motivation is that the different approaches in Table 2 may not differ much in broad statistics about the size of the call graph they produce, such as the number of reachable methods, but they can differ dramatically in the number of paths in the graph. This metric in turn may significantly impact the precision of call graph clients.

We define this client in terms of the reach predicate:

$\operatorname{reach}\left(m, m^{\prime}\right) \Longleftrightarrow \exists c, e, c^{\prime}: \operatorname{method}(e)=m \wedge\left(c, e, c^{\prime}, m^{\prime}\right) \in R$, (where $R=$ leastFix $\lambda X$. (cg $\cup\left\{\left(c, e, c^{\prime \prime}, m\right) \mid \exists c^{\prime}, m^{\prime}, e^{\prime}: \operatorname{method}\left(e^{\prime}\right)=m^{\prime} \wedge\right.$ $\left.\left.\left.\left(c, e, c^{\prime}, m^{\prime}\right) \in X \wedge\left(c^{\prime}, e^{\prime}, c^{\prime \prime}, m\right) \in \mathrm{cg}\right\}\right)\right)$.

The above predicate is true if there exists a path in the context sensitive call graph from $m$ to $m^{\prime}$. The existence of such a path means that it may be possible to invoke $m^{\prime}$ while $m$ is on the call stack, either directly or transitively from a call site in the body $m$. Each query to this client is a pair of methods $\left(m, m^{\prime}\right)$ in the program. This query is proven by an analysis if reach $\left(m, m^{\prime}\right)$ evaluates to false using the call graph cg computed by that analysis - no path exists from $m$ to $m^{\prime}$ in the graph.

Monomorphic Call Inference. Monomorphic call sites are dynamically dispatched call sites with at most one target method. They can be transformed into statically dispatched ones that are cheaper to run. We define a client to statically infer such sites, in terms of the polycall predicate: polycall $(e) \Longleftrightarrow$ $\left|\left\{m \mid \exists c, c^{\prime}:\left(c, e, c^{\prime}, m\right) \in \mathrm{cg}\right\}\right|>1$, where the command at control-flow edge $e$ is a dynamically dispatching call. Each query to this client is a dynamically dispatched call site $e$ in the program. The query is proven by an analysis if polycall $(e)$ evaluates to false using call graph cg computed by that analysis.

We next summarize our evaluation results, including precision, interesting statistics, and scalability of the various approaches on our pointer analysis and its three clients described above. 


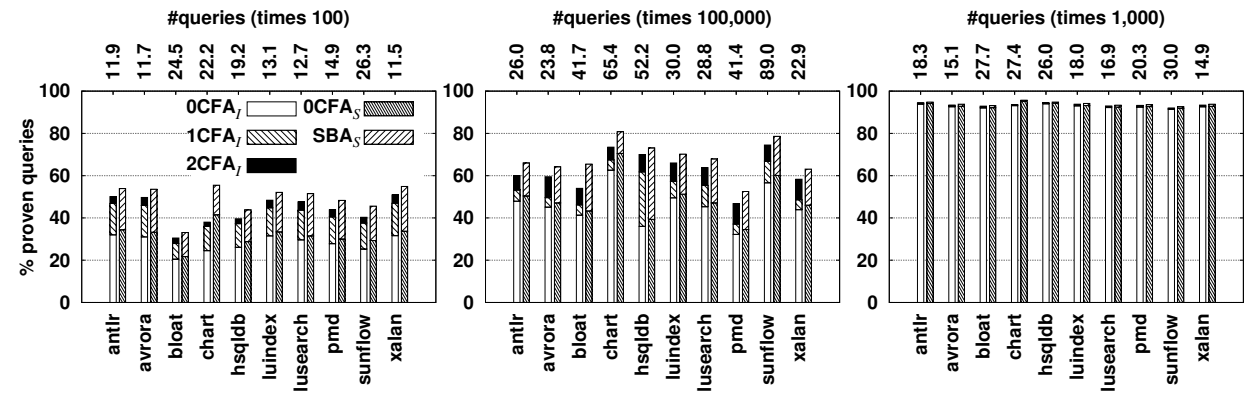

(a) Downcast safety.

(b) Call graph reachability. (c) Monomorphic inference. Fig. 8: Precision of various interprocedural approaches on clients of pointer analysis.

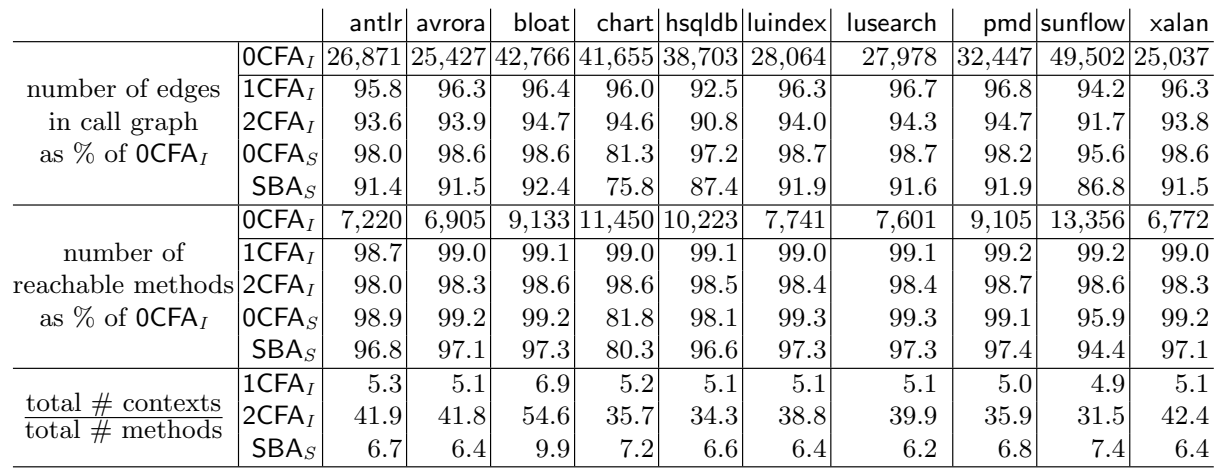

Table 3: Statistics of call graphs computed by various interprocedural approaches.

Precision on Clients. Figure 8 shows the precision of the approaches on the three clients. We measure the precision of an approach on a client in terms of how many queries posed by the client can be proven by the approach on each benchmark. The total number of queries is shown at the top. For instance, for antlr, there are $11.9 \times 10^{2}$ queries by the downcast safety client.

The stacked bars in the plots show the fraction of queries proven by the various approaches. We use separate bars for the flow-insensitive and flow-sensitive approaches, and vary only the degree of context sensitivity within each bar. At the base of each kind of bar is the fraction of queries proven by the context insensitive approaches $\left(\mathrm{CCFA}_{I}\right.$ and $\left.0 \mathrm{CFA} \mathrm{A}_{S}\right)$. The bars stacked above them denote fractions of queries proven exclusively by the indicated context sensitive approaches. For instance, for the downcast safety client on antlr, the left bar shows that 0 CFA $A_{I}$ proves $32 \%$ queries, 1 CFA proves an additional $15 \%$ queries (for a total of $47 \%$ proven queries), and $2 \mathrm{CFA}_{I}$ proves another $3 \%$ queries (for a total of $50 \%$ proven queries). The right bar shows that CCFA $_{S}$ proves $34 \%$ queries, and $\mathrm{SBA}_{S}$ proves an additional $20 \%$ queries (for a total of $54 \%$ proven queries). We next briefly summarize the results.

The $\mathrm{SBA}_{S}$ approach is theoretically the most precise of all five approaches. Compared to the next most precise approach $2 \mathrm{CFA}_{I}$, it proves $12 \%$ more down- 
antlr |avrora bloat chart |hsqldb luindex|lusearch pmd |sunflow xalan

\begin{tabular}{l|r|r|r|r|r|r|r|r|r|r}
\hline 0CFA $_{I}$ & $1 \mathrm{~m} 45 \mathrm{~s}$ & $1 \mathrm{~m} 42 \mathrm{~s}$ & $3 \mathrm{~m} 10 \mathrm{~s}$ & $4 \mathrm{~m} 40 \mathrm{~s}$ & $3 \mathrm{~m} 29 \mathrm{~s}$ & $2 \mathrm{~m} 34 \mathrm{~s}$ & $2 \mathrm{~m} 22 \mathrm{~s}$ & $3 \mathrm{~m} 52 \mathrm{~s}$ & $5 \mathrm{~m} 00 \mathrm{~s}$ & $2 \mathrm{~m} 32 \mathrm{~s}$ \\
1CFA $_{I}$ & $40 \mathrm{~m}$ & $38 \mathrm{~m}$ & $82 \mathrm{~m}$ & $121 \mathrm{~m}$ & $74 \mathrm{~m}$ & $41 \mathrm{~m}$ & $43 \mathrm{~m}$ & $61 \mathrm{~m}$ & $148 \mathrm{~m}$ & $36 \mathrm{~m}$ \\
2CFA $_{I}$ & $72 \mathrm{~m}$ & $68 \mathrm{~m}$ & $239 \mathrm{~m}$ & $256 \mathrm{~m}$ & $158 \mathrm{~m}$ & $83 \mathrm{~m}$ & $80 \mathrm{~m}$ & $112 \mathrm{~m}$ & $279 \mathrm{~m}$ & $82 \mathrm{~m}$ \\
0CFA $_{S}$ & $23 \mathrm{~m}$ & $26 \mathrm{~m}$ & $38 \mathrm{~m}$ & $30 \mathrm{~m}$ & $34 \mathrm{~m}$ & $35 \mathrm{~m}$ & $24 \mathrm{~m}$ & $34 \mathrm{~m}$ & $58 \mathrm{~m}$ & $23 \mathrm{~m}$ \\
SBA $_{S}$ & $21 \mathrm{~m}$ & $17 \mathrm{~m}$ & $60 \mathrm{~m}$ & $51 \mathrm{~m}$ & $37 \mathrm{~m}$ & $27 \mathrm{~m}$ & $16 \mathrm{~m}$ & $29 \mathrm{~m}$ & $72 \mathrm{~m}$ & $16 \mathrm{~m}$ \\
\hline
\end{tabular}

Table 4: Running time of pointer analysis using various approaches.

cast safety queries on average per benchmark, and 9\% more call graph reachability queries, but only $0.6 \%$ more monomorphic call site inference queries. The largest gain of $\mathrm{SBA}_{S}$ over $2 \mathrm{CFA}_{I}$ is $21.3 \%$, and occurs on bloat for the call graph reachability client. The relatively lower benefit of increased context sensitivity for the monomorphic call site inference client is because the context insensitive approaches are themselves able to prove over $90 \%$ of the queries by this client on each benchmark. We also observe that $0 \mathrm{CFA}_{S}$ proves only slightly more queries than OCFA $_{I}$ for each client on each benchmark, suggesting that flow sensitivity is ineffective without an accompanying increase in context sensitivity. In particular, with the exception of chart, OCFA ${ }_{S}$ proves less queries than 1 CFA ${ }_{I}$.

Call Graph Statistics. We found it instructive to study various statistics of the call graphs computed by the different approaches. The first two sets of rows in Table 3 show the number of reachable methods and the number of edges in the call graphs computed by the different approaches. Both decrease with an increase in the precision of the approach, as expected. But the reduction is much smaller compared to that in the number of unproven queries for the call graph reachability client, shown in Figure 8(b). An unproven reach $\left(m, m^{\prime}\right)$ query indicates the presence of one or more paths in the call graph from $m$ to $m^{\prime}$ and the higher the number of such unproven queries, the higher the number of paths in the call graph. The average reduction in the number of such unproven queries from $0 \mathrm{CFA}$ to $\mathrm{SBA}_{S}$ is $41 \%$, but the corresponding average reduction in the number of call graph edges is only $10.8 \%$, and that in the number of reachable methods is even smaller, at $4.8 \%$. From these numbers, we conclude that the various approaches do not differ much in coarse-grained statistics of the call graphs they produce (e.g., the number of reachable methods) but they can differ dramatically in finer-grained statistics (e.g., the number of paths), which in turn can greatly impact the precision of certain clients.

Scalability. Lastly, we compare the scalability of the different approaches. Table 4 shows their running time on our pointer analysis, exclusive of the clients' running time which is negligible. The running time increases from $0 C F A_{I}$ to $2 \mathrm{CFA}_{I}$ with large differences between the different flow insensitive approaches. The similar running times of $0 \mathrm{CFA}{ }_{S}$ and $\mathrm{SBA}_{S}$ is because of the use of the tabulation algorithm with almost identical implementation for both. Finally, $\mathrm{SBA}_{S}$ runs much faster than $2 \mathrm{CFA}_{I}$ on all benchmarks.

The improved performance of $\mathrm{SBA}_{S}$ over $2 \mathrm{CFA}_{I}$ can be explained by the ratio of the number of contexts to that of reachable methods computed by each approach. This ratio is shown in the bottom set of rows in Table 3 for 1 CFA $I$, 
$2 \mathrm{CFA}_{I}$, and $\mathrm{SBA}_{S}$. (It is not shown for context insensitive approaches $0 \mathrm{CFA}$ and $\mathrm{OCFA}_{S}$ as it is the constant 1 for them.) These numbers elicit two key observations. First, the rate at which the ratio increases as we go from 0 CFA $I$ to $2 \mathrm{CFA}_{I}$ suggests that call-strings approaches with $k \geq 3$ run out of memory by computing too many contexts. Second, $2 \mathrm{CFA}_{I}$ computes almost $4 \mathrm{X}-7 \mathrm{X}$ more contexts per method than $\mathrm{SBA}_{S}$ on each benchmark, implying that the summarybased approach used in $\mathrm{SBA}_{S}$ is able to merge many call-string contexts.

The primary purpose of the empirical evaluation in this work was to determine how precise $k$-CFA can get using arbitrary $k$. The proof of equivalence between $\infty$-CFA and SBA enabled us to use $\mathrm{SBA}_{S}$ for this evaluation. However, other works [MRR05, LH08] have shown that, in practice, using object-sensitivity MRR02, SBL11 to distinguish calling contexts for object-oriented programs is more precise and scalable than $k$-CFA. Though call string and object-sensitive contexts are incomparable in theory, an interesting empirical evaluation in future work would be to compare the precision of $\infty$-CFA with analyses using object-sensitive contexts.

\section{Related Work}

This section relates our work to existing equivalence results, work on summarybased approaches, and work on cloning-based approaches of which call-strings approaches are an instance.

Equivalence Results. Sharir and Pnueli SP81] prove that the summary-based and call-strings approaches are equivalent in the finite, distributive setting. They provide constructive algorithms for both approaches in this setting: an iterative fixpoint algorithm for the summary-based approach and an algorithm to obtain a finite bound on the lengths of call strings to be computed for the call-strings approach. They prove each of these algorithms equivalent to the meet-overall-valid-paths (MVP) solution (see Corollary 3.5 and Theorem 5.4 in [SP81]). Their equivalence proof thus relies on the distributivity assumption. Our work can be viewed as an extension of their result to the more general non-distributive setting. Also, they do not provide any empirical results, whereas we measure the precision and scalability of both approaches on a widely-used pointer analysis, using real-world programs and clients.

For points-to analyses, Grove and Chambers GC01 conjectured that Agesen's Cartesian Product Algorithm (CPA) Age95 is strictly more precise than $\infty$-CFA, and that SBA(which they refer as SCS for Simple Class Set)has the same precision as $\infty$-CFA. The first conjecture was shown to be true by Besson Bes09 while we proved that the second conjecture also holds in this work.

Might et al. MSH10 show the equivalence between $k$-CFA in the objectoriented and functional paradigms. The treatment of objects vs. closures in the two paradigms causes the same $k$-CFA algorithm to be polynomial in program size in the object-oriented paradigm but EXPTIME-complete in the functional paradigm. Our work is orthogonal to theirs. Specifically, our formal setting is agnostic to language features, assuming only a finite abstract domain $\boldsymbol{\Gamma}$ and mono- 


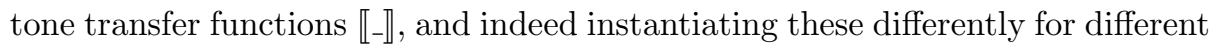
language features can cause the $k$-CFA algorithm to have different complexity.

Summary-based Interprocedural Analysis. Sharir and Pnueli SP81] first proposed using functional summaries to solve interprocedural dataflow problems precisely. Later, Reps et al. RHS95] proposed an efficient quadratic representation of functional summaries for finite, distributive dataflow problems, and the tabulation algorithm based on CFL-reachability to solve them in cubic time. More recent works have applied the tabulation algorithm in non-distributive settings, ranging from doing a fully lossy join to a partial join to a lossless join. All these settings besides lossy join are challenging to scale, and either use symbolic representations (e.g., BDDs in BR01]) to compactly represent multiple abstract states, or share common parts of multiple abstract states without losing precision (e.g., $\mathrm{YLB}^{+} 08$, MSRF04]) or at the expense of precision (e.g., [BPR01]). Summary-based approaches like CFA2 VS10] have also been proposed for functional languages to perform fully context-sensitive control-flow analysis. Our work is motivated by the desire to understand the formal relationship between the widely-used summary-based approach in non-distributive settings and the call-strings approach, which is also prevalent as we survey next.

Cloning-based Interprocedural Analysis. There is a large body of work on bounded call-string-like approaches that we collectively call cloning-based approaches. Besides $k$-CFA [Shi88, another popular approach is $k$-object sensitive analysis for object-oriented programs [MRR02, SBL11. Many recent works express cloning-based pointer analyses in Datalog and solve them using specialized Datalog solvers [Wha07, BS09]. These solvers exploit redundancy arising from large numbers of similar contexts computed by these approaches for high $k$ values. They either use BDDs $\mathrm{BLQ}^{+} 03$, WL04, ZC04 or explicit representations from the databases literature BS09] for this purpose. Most cloning-based approaches approximate recursion in an ad hoc manner. An exception is the work of Khedker et al. KMR12, KK08 which maintains a single representative call string for each equivalence class. Unlike the above approaches, it does not approximate recursion in an ad hoc manner, and yet it is efficient in practice by avoiding the computation of redundant call-string contexts. Our pointer analysis achieves a similar effect but by using the tabulation algorithm.

\section{Conclusion}

We showed the equivalence between the summary-based and unbounded callstrings approaches to interprocedural analysis, in the presence of a lossy join. Our result extends the formal relationship between these approaches to a setting more general than the distributive case in which this result was previously proven. We presented new implications of our result to the theory and practice of interprocedural analysis. On the theoretical side, we introduced new proof techniques that enable to reason about relationships that do not hold between two fixpoint computations at each step, but do so when a form of garbage collection is applied to the final results of those computations. On the practical side, we 
empirically compared the summary-based and bounded call-strings approaches on a widely-used pointer analysis with a lossy join. We found the summary-based approach on this analysis is more scalable while providing the same precision as the unbounded call-strings approach.

Acknowledgement. We thank the anonymous reviewers for insightful comments. This work was supported by DARPA under agreement \#FA8750-12-20020, NSF award \#1253867, gifts from Google and Microsoft, and EPSRC. The U.S. Government is authorized to reproduce and distribute reprints for Governmental purposes notwithstanding any copyright notation thereon.

\section{References}

[Age95] Ole Agesen. The cartesian product algorithm. In ECOOP. 1995.

[Bes09] Frédéric Besson. CPA beats $\infty$-CFA. In FTfJP, 2009.

$\left[\mathrm{BLQ}^{+} 03\right]$ M. Berndl, O. Lhoták, F. Qian, L. Hendren, and N. Umanee. Points-to analysis using BDDs. In PLDI, 2003.

[BPR01] T. Ball, A. Podelski, and S. Rajamani. Boolean and cartesian abstraction for model checking C programs. In $T A C A S, 2001$.

[BR01] T. Ball and S. Rajamani. Bebop: a path-sensitive interprocedural dataflow engine. In PASTE, 2001.

[BS09] M. Bravenboer and Y. Smaragdakis. Strictly declarative specification of sophisticated points-to analyses. In OOPSLA, 2009.

[CC79] P. Cousot and R. Cousot. Systematic design of program analysis frameworks. In $P O P L$, 1979

[CC92] P. Cousot and R. Cousot. Abstract interpretation frameworks. Journal of Logic and Computation, 2(4), 1992

$\left[\mathrm{FYD}^{+} 08\right]$ S. Fink, E. Yahav, N. Dor, G. Ramalingam, and E. Geay. Effective typestate verification in the presence of aliasing. ACM TOSEM, 17(2), 2008.

[GC01] David Grove and Craig Chambers. A framework for call graph construction algorithms. ACM TOPLAS, 23(6), 2001.

[KK08] U. Khedker and B. Karkare. Efficiency, precision, simplicity, and generality in interprocedural dataflow analysis: Resurrecting the classical call strings method. In $C C, 2008$.

[KMR12] U. Khedker, A. Mycroft, and P. Rawat. Liveness-based pointer analysis. In SAS, 2012.

[LH08] O. Lhoták and L. Hendren. Evaluating the benefits of context-sensitive points-to analysis using a BDD-based implementation. ACM TOSEM, 18(1), 2008.

[Min06] A. Miné. The octagon abstract domain. Higher-Order and Symbolic Computation, 19(1), 2006.

[MRR02] A. Milanova, A. Rountev, and B. Ryder. Parameterized object sensitivity for points-to and side-effect analyses for Java. In ISSTA, 2002.

[MRR05] Ana Milanova, Atanas Rountev, and Barbara G. Ryder. Parameterized object sensitivity for points-to analysis for Java. ACM TOSEM, 14(1), 2005.

[MSH10] M. Might, Y. Smaragdakis, and D. Horn. Resolving and exploiting the $k$-CFA paradox: illuminating functional vs. oo program analysis. In PLDI, 2010.

[MSRF04] R. Manevich, M. Sagiv, G. Ramalingam, and J. Field. Partially disjunctive heap abstraction. In $S A S, 2004$.

[RHS95] T. Reps, S. Horwitz, and M. Sagiv. Precise interprocedural dataflow analysis via graph reachability. In POPL, 1995.

[SBL11] Y. Smaragdakis, M. Bravenboer, and O. Lhoták. Pick your contexts well: understanding object-sensitivity. In POPL, 2011.

[Shi88] O. Shivers. Control-flow analysis in scheme. In PLDI, 1988.

[SP81] M. Sharir and A. Pnueli. Two approaches to interprocedural data flow analysis. In Program Flow Analysis: Theory and Applications, chapter 7. Prentice-Hall, 1981.

[VS10] D. Vardoulakis and O. Shivers. CFA2: A Context-Free Approach to Control-Flow Analysis. In ESOP, 2010.

[Wha07] J. Whaley. Context-Sensitive Pointer Analysis using Binary Decision Diagrams. PhD thesis, Stanford University, March 2007.

[WL04] J. Whaley and M. Lam. Cloning-based context-sensitive pointer alias analysis using binary decision diagrams. In PLDI, 2004.

$\left[\mathrm{YLB}^{+} 08\right]$ H. Yang, O. Lee, J. Berdine, C. Calcagno, B. Cook, D. Distefano, and P. O'Hearn. Scalable shape analysis for systems code. In $C A V, 2008$.

[ZC04] J. Zhu and S. Calman. Symbolic pointer analysis revisited. In PLDI, 2004. 


\section{Appendix}

\section{A Proofs of Lemmas}

\section{A.1 Lemma 1}

Lemma 1. For all $\sigma$ and $\kappa$, if $\sigma \sqsubseteq R(\eta, \kappa)$, then $L(\eta, \sigma) \sqsubseteq \kappa$.

Proof. Pick any $\sigma$ and $\kappa$. Suppose that $\sigma \sqsubseteq R(\eta, \kappa)$. Then, $\sigma(p, \tau) \sqsubseteq R(\eta, \kappa)(p, \tau)$ for all $p, \tau$. By the definition of $R$, this means that for all $p, \tau, \pi$, if $\tau \sqsubseteq \eta(p, \pi)$, then $\sigma(p, \tau) \sqsubseteq \kappa(p, \pi)$. By setting $\tau=\eta(p, \pi)$, we can derive from this implication the formula: $\forall p, \pi: \sigma(p, \eta(p, \pi)) \sqsubseteq \kappa(p, \pi)$. We rephrase this formula using $L$ : $\forall p, \pi: L(\eta, \sigma)(p, \pi) \sqsubseteq \kappa(p, \pi)$. The rephrase formula gives the desired conclusion $L(\eta, \sigma) \sqsubseteq \kappa$.

Remark 1. The opposite direction of Lemma 1 holds only if $\sigma$ is monotone in the following sense: for all $p, \tau, \tau^{\prime}$, if $\tau \sqsubseteq \tau^{\prime}$, then $\sigma(p, \tau) \sqsubseteq \sigma\left(p, \tau^{\prime}\right)$. In fact, there is a function $R^{\prime}(\eta,-): \mathbf{A}_{\mathrm{cfa}} \rightarrow \mathbf{A}_{\mathrm{sba}}$ that gives the equivalence without this proviso on monotonicity: $R^{\prime}(\eta, \kappa)=\lambda(p, \tau) . \prod\{\kappa(p, \pi) \mid \tau=\eta(\operatorname{method}(p), \pi)\}$. We do not use $R^{\prime}$ because $R(\eta, \kappa)$ is always monotone but $R^{\prime}(\eta, \kappa)$ is not. This monotonicity is exploited in our proof of our Correspondence Theorem.

\section{A.2 Lemma 2}

Lemma 4. $\sigma_{\text {sba }}(p, \perp)=\perp$ for all $p \in \mathbf{P}$.

Proof. This lemma holds because $\sigma_{I}$ and the smallest SBA annotation

$$
\lambda(p, \tau) \cdot \perp
$$

are strict, and both $\sqcup$ and $F_{\text {sba }}$ preserve strictness: if $\sigma$ and $\sigma^{\prime}$ are strict, so are $\sigma \cup \sigma^{\prime}$ and $F_{\text {sba }}(\sigma)$.

Lemma 2. There exists a unique translation function $\eta: \mathbf{M} \times \mathbf{P} \rightarrow \boldsymbol{\Gamma}$ such that for all $m \in \mathbf{M}, e \in \mathbf{E}$ and $\pi \in \mathbf{\Pi}$,

$$
\begin{aligned}
& \eta\left(m_{\text {main }}, m_{\text {main }}\right)=\tau_{\text {init }}, \\
& \eta(m, m \oplus e \oplus \pi)= \\
& \text { if }(\operatorname{callEdge}(e) \wedge \\
& \left.\quad m \in \operatorname{calls}\left(\operatorname{stmt}(e), \sigma_{\text {sba }}(\operatorname{origin}(e), \eta(\operatorname{method}(e), \pi))\right)\right) \\
& \text { then } \sigma_{\text {sba }}(\operatorname{origin}(e), \eta(\text { method }(e), \pi)) \text { else } \perp, \\
& \eta(m, \pi)=\perp \quad(\text { for all the other cases }) .
\end{aligned}
$$

We denote this translation with $\eta_{\text {sba }}$. 
Proof. First, we show the existence. Let $\eta_{\text {sba }}$ be the least upper bound of a sequence $\left\{\eta_{n}\right\}_{n \geq 0}$ :

$$
\eta(m, \pi)=\bigsqcup\left\{\eta_{n}(m, \pi) \mid n \geq 0\right\}
$$

where $\eta_{0}$ is the constant map $\lambda(p, \pi) . \perp$ and $\eta_{n+1}$ is defined by:

$$
\begin{aligned}
& \eta_{n+1}\left(m_{\text {main }}, m_{\text {main }}\right)=\tau_{\text {init }} \\
& \eta_{n+1}(m, m \oplus e \oplus \pi)= \\
& \text { if }(\operatorname{callEdge}(e) \wedge \\
& \left.\quad m \in \operatorname{calls}\left(\operatorname{stmt}(e), \sigma_{\text {sba }}\left(\operatorname{origin}(e), \eta_{n}(\operatorname{method}(e), \pi)\right)\right)\right) \\
& \text { then } \sigma_{\mathrm{sba}}\left(\operatorname{origin}(e), \eta_{n}(\operatorname{method}(e), \pi)\right) \text { else } \perp \\
& \eta_{n+1}(m, \pi)=\perp \quad(\text { for all the other cases }) .
\end{aligned}
$$

We will show that $\eta$ satisfies the properties described in the lemma. Since $\eta_{n}\left(m_{\text {main }}, m_{\text {main }}\right)=\tau_{\text {init }}$ for all $n \geq 1$ and $\eta_{0}\left(m_{\text {main }}, m_{\text {main }}\right)=\perp$,

$$
\eta_{\text {sba }}\left(m_{\text {main }}, m_{\text {main }}\right)=\tau_{\text {init }} .
$$

For the other properties, we will show that

$$
\forall n, m, \pi: \eta_{n}(m, \pi) \neq \perp \Longrightarrow \forall k \geq n: \eta_{k}(m, \pi)=\eta_{n}(m, \pi) .
$$

Before presenting the proof of this formula, let us explain why the formula gives the desired properties of $\eta$. Our starting point is the second property. Suppose that the formula in $(18)$ is true. Then,

$$
\forall m, \pi: \exists n: \forall k \geq n: \eta(m, \pi)=\eta_{k}(m, \pi) .
$$

Now pick $e \in \mathbf{E}, \pi \in \boldsymbol{\Pi}$ and $m \in \mathbf{M}$ such that

$$
\begin{aligned}
& \text { callEdge }(e) \wedge \\
& m \in \operatorname{calls}\left(\operatorname{stmt}(e), \sigma_{\mathrm{sba}}(\operatorname{origin}(e), \eta(\operatorname{method}(e), \pi))\right) .
\end{aligned}
$$

By what we have just discussed, there exists a non-negative integer $n$ such that

$$
\begin{aligned}
& \forall k \geq n: \eta(\operatorname{method}(e), \pi)=\eta_{k}(\operatorname{method}(e), \pi) \wedge \\
& \eta(m, m \oplus e \oplus \pi)=\eta_{k}(m, m \oplus e \oplus \pi) .
\end{aligned}
$$

Since $\eta(\operatorname{method}(e), \pi)=\eta_{n}(\operatorname{method}(e), \pi)$,

$$
m \in \operatorname{calls}\left(\operatorname{stmt}(e), \sigma_{\mathrm{sba}}\left(\operatorname{origin}(e), \eta_{n}(\operatorname{method}(e), \pi)\right)\right) .
$$

By the definition of $\eta_{n+1}$, we have that

$$
\eta_{n+1}(m, m \oplus e \oplus \pi)=\sigma_{\text {sba }}\left(\operatorname{origin}(e), \eta_{n}(\operatorname{method}(e), \pi)\right) .
$$

Using (19), we can conclude that

$$
\eta(m, m \oplus e \oplus \pi)=\sigma_{\text {sba }}(\operatorname{origin}(e), \eta(\operatorname{method}(e), \pi)),
$$


which is the desired equality. We also have to consider the case that $e \in \mathbf{E}$, $\pi \in \boldsymbol{\Pi}$ and $m \in \mathbf{M}$ but the following does not hold:

$$
\begin{aligned}
& \text { callEdge }(e) \wedge \\
& m \in \operatorname{calls}\left(\operatorname{stmt}(e), \sigma_{\text {sba }}(\operatorname{origin}(e), \eta(\operatorname{method}(e), \pi))\right) .
\end{aligned}
$$

As before, let $n$ be the same value satisfying (19). Then, for all $k \geq n$, the following statement does not hold:

$$
\begin{aligned}
& \text { calledge }(e) \wedge \\
& m \in \operatorname{calls}\left(\operatorname{stmt}(e), \sigma_{\text {sba }}\left(\operatorname{origin}(e), \eta_{k}\left(\operatorname{method}_{k}(e), \pi\right)\right)\right) .
\end{aligned}
$$

Hence, $\eta_{k+1}(m, m \oplus e \oplus \pi)=\perp$. This implies that

$$
\eta(m, m \oplus e \oplus \pi)=\perp
$$

as well. For the proof of the third property, pick $m, \pi$ such that

$$
(m, \pi) \neq\left(m_{\text {main }}, m_{\text {main }}\right) \wedge \neg \exists e, \pi^{\prime}:(m, \pi) \neq\left(m, m \oplus e \oplus \pi^{\prime}\right) .
$$

Then, $\eta_{n}(m, \pi)=\perp$ for all $n$. Hence, $\eta(m, \pi)=\perp$.

Let us now go back to the proof of (18). Our proof is by the induction on $n$ in $\eta_{n}$.

1. Base case $n=0$ : In this case, $\eta_{n}(m, \pi)=\perp$ for all $m$ and $\pi$. Hence, (18) follows vacuously.

2. Inductive case $n>0$ : Assume that

$$
\eta_{n}(m, \pi) \neq \perp
$$

By the definition of $\eta_{n}$, this can happen only under two cases: (i) $\pi=m_{\text {main }}$ and $m=m_{\text {main }}$; or (ii) $\pi=m \oplus e \oplus \pi_{1}$ for some $e, \pi_{1}$ such that

$$
\begin{aligned}
& \text { callEdge }(e) \wedge \\
& m \in \operatorname{calls}\left(\operatorname{stmt}(e), \sigma_{\text {sba }}\left(\operatorname{origin}(e), \eta_{n-1}\left(\operatorname{method}(e), \pi_{1}\right)\right)\right) .
\end{aligned}
$$

If $\pi=m_{\text {main }}$ and $m=m_{\text {main }}$,

$$
\forall k \geq 1: \eta_{k}(m, \pi)=\tau_{\text {init }} .
$$

This gives the desired conclusion. Now consider the other case. Let $e, \pi_{1}$ be the entities satisfying (20). Since

$$
\operatorname{calls}(\operatorname{stmt}(e), \perp)=\emptyset,
$$

the second conjunct of 20 implies that

$$
\sigma_{\mathrm{sba}}\left(\operatorname{origin}(e), \eta_{n-1}\left(\operatorname{method}(e), \pi_{1}\right)\right) \neq \perp .
$$


By Lemma 4.

$$
\eta_{n-1}\left(\operatorname{method}(e), \pi_{1}\right) \neq \perp \text {. }
$$

Then, by the induction hypothesis,

$$
\forall k \geq n-1: \eta_{n-1}\left(\operatorname{method}(e), \pi_{1}\right)=\eta_{k}\left(\operatorname{method}(e), \pi_{1}\right) .
$$

This implies that

$$
\begin{aligned}
& \forall k \geq n: \\
& \quad m \in \operatorname{calls}\left(\operatorname{stmt}(e), \sigma_{\text {sba }}\left(\operatorname{origin}(e), \eta_{k-1}\left(\operatorname{method}(e), \pi_{1}\right)\right)\right) .
\end{aligned}
$$

Using this formula, we infer that for all $k \geq n$,

$$
\begin{aligned}
& \eta_{k}\left(m, m \oplus e \oplus \pi_{1}\right) \\
& =\sigma_{\text {sba }}\left(\operatorname{origin}(e), \eta_{k-1}\left(\operatorname{method}(e), \pi_{1}\right)\right) \\
& =\sigma_{\text {sba }}\left(\operatorname{origin}(e), \eta_{n-1}\left(\operatorname{method}(e), \pi_{1}\right)\right) \\
& =\eta_{n}\left(m, m \oplus e \oplus \pi_{1}\right) .
\end{aligned}
$$

We have just shown the desired equality.

Next, we prove the uniqueness. This can be easily proved by induction on the length of $\pi$ in $\eta(m, \pi)$. This is because the conditions in the lemma are inductive on $\pi$ in the sense that the defining clauses of $\eta(m, \pi)$ either do not mention $\pi$ or use $\eta\left(m, \pi^{\prime}\right)$ with strictly shorter suffices $\pi^{\prime}$ of $\pi$.

\section{A.3 Lemma 3}

Lemma 3. For all SBA annotations $\sigma$, if $\sigma$ is monotone, $G_{\text {sba }}(\sigma) \sqsubseteq \sigma$ and

$$
\forall m: \tau \sqsubseteq \sigma(\operatorname{entry}(m), \tau),
$$

then $\sigma_{\text {sba }} \sqsubseteq \sigma$.

Proof. Let $G=\left(\lambda \sigma . \sigma \sqcup F_{\text {sba }}(\sigma)\right)$. Recall that $\sigma_{\text {sba }}$ is defined as the fixpoint (or stable element) of the sequence $G^{0}\left(\sigma_{I}\right), G^{1}\left(\sigma_{I}\right), G^{2}\left(\sigma_{I}\right), G^{3}\left(\sigma_{I}\right), \ldots$ Let $L^{n}=$ $G^{n}\left(\sigma_{I}\right)$. We will prove that for all $n \geq 0$,

$$
L^{n} \sqsubseteq \sigma .
$$

Our proof is by induction on $n$. When $n=0$, our assumption gives $L^{0}=\sigma_{I} \sqsubseteq \sigma$, as desired. Assume that $n>0$. By induction hypothesis, we have that $L^{n-1} \sqsubseteq \sigma$. This means that to prove $L^{n} \sqsubseteq \sigma$, it is sufficient to show that

$$
F_{\text {sba }}\left(L^{n-1}\right) \sqsubseteq \sigma .
$$

Let $p \in \mathbf{P}$ and $\tau \in \boldsymbol{\Gamma}$. When $p$ is a non-entry node or a non-call edge, the desired

$$
F_{\mathrm{sba}}\left(L^{n-1}\right)(p, \tau) \sqsubseteq \sigma(p, \tau)
$$


follows from the monotonicity of $\sqcup$ and $\llbracket s \rrbracket$ for every statement $s \in \mathbf{A} \cup \mathbf{I}$, and the facts that $F_{\text {sba }}(\sigma)(p, \tau) \sqsubseteq \sigma(p, \tau)$ and $L^{n-1} \sqsubseteq \sigma$. When $p$ is an entry node of a method, $F_{\text {sba }}\left(L^{n-1}\right)(p, \tau)$ is either $\perp$ or $\tau$. In both cases, $F_{\text {sba }}\left(L^{n-1}\right)(p, \tau) \sqsubseteq$ $\sigma(p, \tau)$, because $\tau^{\prime} \sqsubseteq \sigma\left(\operatorname{entry}(m), \tau^{\prime}\right)$ for every $m$ and $\tau^{\prime}$. The only remaining case is that $p$ is a call edge. In this case, $F_{\text {sba }}\left(L^{n-1}\right)(p, \tau)$ is defined as the join of a set described at the bottom of Figure 5 (modulo the replacement of $e$ and $\sigma$ by $p$ and $L^{n-1}$ in the figure). Let $\Gamma$ be this set. Similarly, $F_{\text {sba }}(\sigma)(p, \tau)$ is defined as the join of a similar set $\Gamma^{\prime}$. It is sufficient to show that

$$
\forall \tau^{\prime} \in \Gamma: \exists \tau^{\prime \prime} \in \Gamma^{\prime}: \tau^{\prime} \sqsubseteq \tau^{\prime \prime} .
$$

Pick $\tau^{\prime}$ from $\Gamma$. Then, there exist $\tau_{1}, m$ such that $\tau_{1}=L^{n-1}(\operatorname{origin}(p), \tau)$, $m \in \operatorname{calls}\left(\operatorname{stmt}(p), \tau_{1}\right)$ and $\tau^{\prime}=L^{n-1}\left(\operatorname{exit}(m), \tau_{1}\right)$. Since $L^{n-1} \sqsubseteq \sigma$ and calls is monotone, there exists $\tau_{1}^{\prime}$ such that $\tau_{1} \sqsubseteq \tau_{1}^{\prime}, \tau_{1}^{\prime}=\sigma(\operatorname{origin}(p), \tau)$ and $m \in$ calls $\left(\operatorname{stmt}(p), \tau_{1}^{\prime}\right)$. Furthermore, since $L^{n-1} \sqsubseteq \sigma$ and $\sigma$ is monotone,

$$
\tau^{\prime}=L^{n-1}\left(\operatorname{exit}(m), \tau_{1}\right) \sqsubseteq \sigma\left(\operatorname{exit}(m), \tau_{1}\right) \sqsubseteq \sigma\left(\operatorname{exit}(m), \tau_{1}^{\prime}\right) .
$$

Let $\tau^{\prime \prime}=\sigma\left(\operatorname{exit}(m), \tau_{1}^{\prime}\right)$. What we have shown so far implies $\tau^{\prime \prime} \in \Gamma^{\prime} \wedge \tau^{\prime \prime} \sqsupseteq \tau^{\prime}$.

\section{B Proof of the Correspodence Theorem}

In this section, we present the full proof of the Correspondence Theorem (Theorem 2 .

\section{B.1 Step One}

The aim of this subsection is to prove the following proposition.

Proposition 1. $\kappa_{\mathrm{cfa}} \sqsubseteq L\left(\eta_{\mathrm{sba}}, \sigma_{\mathrm{sba}}\right)$.

To achieve our aim, we will develop a method for transferring pre-fixpoints of $\lambda \sigma . \sigma_{I} \sqcup F_{\text {sba }}(\sigma)$ to those of $\lambda \kappa . \kappa_{I} \sqcup F_{\text {cfa }}(\kappa)$. Concrete, we identify a sufficient condition on a translation function $\eta$ for ensuring that the result of the $\infty$ CFA analysis is at least as precise as that of the SBA analysis if the latter gets translated by $L(\eta,-)$. Our condition is based on the following two lemmas, which together express which $\eta$ allows transferring correct SBA annotations (i.e., prefixpoints with respect to $\left.\lambda \sigma . \sigma_{I} \sqcup F_{\text {sba }}(\sigma)\right)$ to correct $\infty$-CFA annotations (i.e., pre-fixpoints with respect to $\left.\lambda \kappa . \kappa_{I} \sqcup F_{\text {cfa }}(\kappa)\right)$.

Lemma 5. For all translation functions $\eta: \mathbf{P} \times \boldsymbol{\Pi} \rightarrow \boldsymbol{\Gamma}$,

$$
\tau_{\text {init }}=\eta\left(m_{\text {main }}, m_{\text {main }}\right) \Longrightarrow \kappa_{I} \sqsubseteq L\left(\eta, \sigma_{I}\right) .
$$

Proof. Let $n_{0}=\operatorname{entry}\left(m_{\text {main }}\right)$ and $\pi_{0}=m_{\text {main }}$. Recall that $\kappa_{I}(n, \pi)=\perp$ unless $(n, \pi)=\left(n_{0}, \pi_{0}\right)$. Hence, it suffices to show that $\kappa_{I}\left(n_{0}, \pi_{0}\right) \sqsubseteq L\left(\eta, \sigma_{I}\right)\left(n_{0}, \pi_{0}\right)$. We discharge this proof obligation as follows:

$$
\begin{aligned}
\tau_{\text {init }}=\eta\left(m_{\text {main }}, \pi_{0}\right) & \Longleftrightarrow \tau_{\text {init }}=\sigma_{I}\left(n_{0}, \eta\left(m_{\text {main }}, \pi_{0}\right)\right) \\
& \Longleftrightarrow \kappa_{I}\left(n_{0}, \pi_{0}\right)=L\left(\eta, \sigma_{I}\right)\left(n_{0}, \pi_{0}\right) .
\end{aligned}
$$


A translation $\eta$ is consistent with an SBA annotation $\sigma$ if for all $e \in \mathbf{E}$, $\pi \in \boldsymbol{\Pi}$ and $m \in \mathbf{M}$ such that

$$
\begin{aligned}
& \text { callEdge }(e) \\
& \wedge m \in \operatorname{calls}(\operatorname{stmt}(e), \sigma(\operatorname{origin}(e), \eta(\operatorname{method}(e), \pi))),
\end{aligned}
$$

we have

$$
\eta(m, m \oplus e \oplus \pi)=\sigma(\operatorname{origin}(e), \eta(\operatorname{method}(e), \pi)) .
$$

Lemma 6. If a translation $\eta$ is consistent with an $S B A$ annotation $\sigma$,

$$
F_{\text {cfa }}(L(\eta, \sigma)) \sqsubseteq L\left(\eta, F_{\text {sba }}(\sigma)\right) .
$$

Proof. Assume that a translation $\eta$ is consistent with an SBA annotation $\sigma$. Pick $p, \pi$. We will show that

$$
F_{\text {cfa }}(L(\eta, \sigma))(p, \pi) \sqsubseteq L\left(\eta, F_{\text {sba }}(\sigma)\right)(p, \pi) .
$$

We do the case analysis on $p$.

1. The first case is that $p$ is a node $n$ but not an entry node of a method. In this case,

$$
\begin{aligned}
& F_{\mathrm{cfa}}(L(\eta, \sigma))(n, \pi) \\
& =\bigsqcup\{L(\eta, \sigma)(e, \pi) \mid n=\operatorname{target}(e)\} \\
& =\bigsqcup\{\sigma(e, \eta(\operatorname{method}(e), \pi)) \mid n=\operatorname{target}(e)\} .
\end{aligned}
$$

Also,

$$
\begin{aligned}
& L\left(\eta, F_{\mathrm{sba}}(\sigma)\right)(n, \pi) \\
& =F_{\mathrm{sba}}(\sigma)(n, \eta(\operatorname{method}(n), \pi)) \\
& =\bigsqcup\{\sigma(e, \eta(\operatorname{method}(n), \pi)) \mid n=\operatorname{target}(e)\} .
\end{aligned}
$$

The desired equality holds because

$$
n=\operatorname{target}(e) \Longrightarrow \operatorname{method}(n)=\operatorname{method}(e) .
$$

2. The next case is that $p$ is a entry node entry $(m)$. Let $P\left(e, \pi_{1}\right)$ be the predicate that asserts the following:

$$
\begin{aligned}
& \text { callEdge }(e) \wedge \pi=m \oplus e \oplus \pi_{1} \\
& \wedge m \in \operatorname{calls}\left(\operatorname{stmt}(e), L(\eta, \sigma)\left(\operatorname{origin}(e), \pi_{1}\right)\right) .
\end{aligned}
$$

If we cannot find $e, \pi_{1}$ satisfying $P\left(e, \pi_{1}\right)$, then

$$
F_{\text {cfa }}(L(\eta, \sigma))(\operatorname{entry}(m), \pi)=\perp,
$$


which implies the desired inequality in (21). Now assume that $P\left(e, \pi_{1}\right)$ holds for some $e$ and $\pi_{1}$. Then,

$$
\begin{aligned}
& F_{\mathrm{cfa}}(L(\eta, \sigma))(\operatorname{entry}(m), \pi) \\
& =L(\eta, \sigma)\left(\operatorname{origin}(e), \pi_{1}\right) \\
& =\sigma\left(\operatorname{origin}(e), \eta\left(\operatorname{method}(\operatorname{origin}(e)), \pi_{1}\right)\right) \\
& =\sigma\left(\operatorname{origin}(e), \eta\left(\operatorname{method}(e), \pi_{1}\right)\right) .
\end{aligned}
$$

Here the first equality uses the definition of $F_{\mathrm{cfa}}$ and the property in 222 , and the second equality is the unrolling of the definition of $L$. Also, in this case,

$$
\begin{aligned}
& L\left(\eta, F_{\text {sba }}(\sigma)\right)(\operatorname{entry}(m), \pi) \\
& =F_{\text {sba }}(\sigma)(\operatorname{entry}(m), \eta(m, \pi)) \\
& =\bigsqcup\{\eta(m, \pi) \mid \\
& \quad \exists e, \tau_{1}: \eta(m, \pi)=\sigma\left(\operatorname{origin}(e), \tau_{1}\right) \\
& \quad \wedge m \in \operatorname{calls}(\operatorname{stmt}(e), \eta(m, \pi)) \wedge \operatorname{callEdge}(e)\} .
\end{aligned}
$$

Hence, to prove

$$
F_{\text {cfa }}(L(\eta, \sigma))(\operatorname{entry}(m), \pi) \sqsubseteq L\left(\eta, F_{\text {sba }}(\sigma)\right)(\operatorname{entry}(m), \pi),
$$

it suffices to show

$$
\eta(m, \pi)=\sigma\left(\operatorname{origin}(e), \eta\left(\operatorname{method}(e), \pi_{1}\right)\right) .
$$

Since $\pi=m \oplus e \oplus \pi_{1}$, the above equation is equivalen to

$$
\eta\left(m, m \oplus e \oplus \pi_{1}\right)=\sigma\left(\operatorname{origin}(e), \eta\left(\operatorname{method}(e), \pi_{1}\right)\right) .
$$

This equality follows from the assumption that $\eta$ is consistent with $\sigma$.

3. The third case is that $p$ is a non-call edge $e$ (i.e., $\neg$ callEdge $(e)$ ). In this case,

$$
\begin{aligned}
& F_{\mathrm{cfa}}(L(\eta, \sigma))(e, \pi) \\
& =\llbracket \operatorname{stmt}(e) \rrbracket(L(\eta, \sigma)(\operatorname{origin}(e), \pi)) \\
& =\llbracket \operatorname{stmt}(e) \rrbracket(\sigma(\operatorname{origin}(e), \eta(\operatorname{method}(\operatorname{origin}(e)), \pi))) \\
& =\llbracket \operatorname{stmt}(e) \rrbracket(\sigma(\operatorname{origin}(e), \eta(\operatorname{method}(e), \pi))) .
\end{aligned}
$$

Also,

$$
\begin{aligned}
& L\left(\eta, F_{\mathrm{sba}}(\sigma)\right)(e, \pi) \\
& =F_{\mathrm{sba}}(\sigma)(e, \eta(\operatorname{method}(e), \pi)) \\
& =\llbracket \operatorname{stmt}(e) \rrbracket(\sigma(\operatorname{origin}(e), \eta(\operatorname{method}(e), \pi))) .
\end{aligned}
$$

We have just shown that

$$
F_{\text {cfa }}(L(\eta, \sigma))(e, \pi)=L\left(\eta, F_{\text {sba }}(\sigma)\right)(e, \pi),
$$

a fact stronger than what we are required to show here. 
4. The last case is that $p$ is a call edge $e$ (i.e., callEdge $(e)$ ). Let $\kappa=L(\eta, \sigma)$. Then,

$$
\begin{aligned}
& F_{\mathrm{cfa}}(L(\eta, \sigma))(e, \pi) \\
& =\bigsqcup\{L(\eta, \sigma)(\operatorname{exit}(m), m \oplus e \oplus \pi) \mid \\
& \quad m \in \operatorname{calls}(\operatorname{stmt}(e), L(\eta, \sigma)(\operatorname{origin}(e), \pi))\} \\
& =\bigsqcup\{\sigma(\operatorname{exit}(m), \eta(m, m \oplus e \oplus \pi)) \mid \\
& \quad m \in \operatorname{calls}(\operatorname{stmt}(e), \sigma(\operatorname{origin}(e), \eta(\operatorname{method}(e), \pi)))\} \\
& =\bigsqcup\{\sigma(\operatorname{exit}(m), \sigma(\operatorname{origin}(e), \eta(\operatorname{method}(e), \pi))) \mid \\
& \quad m \in \operatorname{calls}(\operatorname{stmt}(e), \sigma(\operatorname{origin}(e), \eta(\operatorname{method}(e), \pi)))\} \\
& =F_{\mathrm{sba}}(\sigma)(e, \eta(\operatorname{method}(e), \pi)) \\
& =L\left(\eta, F_{\mathrm{sba}}(\sigma)\right)(e, \pi) .
\end{aligned}
$$

The third equality holds because $\eta$ is consistent with $\sigma$ and $e$ is a call edge.

Lemma 7. $\eta_{\text {sba }}$ is consistent with $\sigma_{\text {sba }}$ and

$$
\tau_{\text {init }}=\eta_{\text {sba }}\left(\operatorname{entry}\left(m_{\text {main }}\right), m_{\text {main }}\right) .
$$

Proof. This is an immediate consequence of Lemma 2.

Proposition 1, $\kappa_{\text {cfa }} \sqsubseteq L\left(\eta_{\text {sba }}, \sigma_{\text {sba }}\right)$.

Proof. Since $\sigma_{\text {sba }}$ is the result of the SBA analysis,

$$
\sigma_{I} \sqsubseteq \sigma_{\text {sba }} \wedge F_{\text {sba }}\left(\sigma_{\text {sba }}\right) \sqsubseteq \sigma_{\text {sba }} .
$$

By Lemma 7, $\eta_{\text {sba }}$ is consistent with $\sigma_{\text {sba }}$ and

$$
\tau_{\text {init }}=\eta_{\text {sba }}\left(\operatorname{entry}\left(m_{\text {main }}\right), m_{\text {main }}\right) .
$$

Hence, by Lemma 5 and the monotonicity of $L\left(\eta_{\mathrm{sba}},-\right)$,

$$
\kappa_{I} \sqsubseteq L\left(\eta_{\mathrm{sba}}, \sigma_{I}\right) \sqsubseteq L\left(\eta_{\mathrm{sba}}, \sigma_{\mathrm{sba}}\right) .
$$

Also, by Lemma 6 and the monotonicity of $L\left(\eta_{\mathrm{sba}},-\right)$,

$$
F_{\text {cfa }}\left(L\left(\eta, \sigma_{\text {sba }}\right)\right) \sqsubseteq L\left(\eta, F_{\text {sba }}\left(\sigma_{\text {sba }}\right)\right) \sqsubseteq L\left(\eta, \sigma_{\text {sba }}\right) .
$$

We have just shown that $L\left(\eta, \sigma_{\text {sba }}\right)$ is a pre-fixpoint of $\lambda \kappa . \kappa_{I} \sqcup F_{\text {cfa }}(\kappa)$. Since $\kappa_{\text {cfa }}$ is the least pre-fixpoint, we have

$$
\kappa_{\mathrm{cfa}} \sqsubseteq L\left(\eta, \sigma_{\mathrm{sba}}\right) .
$$




\section{B.2 Step Two}

The purpose of this subsection is to show the following proposition:

Proposition 2. $\sigma_{\mathrm{sba}} \sqsubseteq R\left(\eta_{\mathrm{cfa}}, \kappa_{\mathrm{cfa}}\right)$.

As in the previous subsection, we develop a sufficient condition that $R(\eta, \kappa)$ satisfies all the three conditions in (3). Then, we will show that this condition holds for $\eta_{\text {cfa }}$ and $\kappa_{\text {cfa }}$.

Lemma 8. For all translations $\eta: \mathbf{P} \times \mathbf{\Pi} \rightarrow \mathbf{\Gamma}$, if

$$
\begin{aligned}
& \eta\left(m_{\text {main }}, m_{\text {main }}\right)=\tau_{\text {init }} \\
& \wedge\left(\forall \pi: \pi \neq m_{\text {main }} \Longrightarrow \eta\left(m_{\text {main }}, \pi\right)=\perp\right)
\end{aligned}
$$

then

$$
\sigma_{I} \sqsubseteq R\left(\eta, \kappa_{I}\right) .
$$

Proof. Consider $\eta$ satisfying the assumption of the lemma. Let $n_{0}=\operatorname{entry}\left(m_{\text {main }}\right)$. Recall that $\sigma_{I}(n, \tau)=\perp$ unless $(n, \tau)=\left(n_{0}, \tau_{\text {init }}\right)$. Hence, it suffices to show that

$$
\sigma_{I}\left(n_{0}, \tau_{\text {init }}\right) \sqsubseteq R\left(\eta, \kappa_{I}\right)\left(n_{0}, \tau_{\text {init }}\right),
$$

which is equivalent to

$$
\tau_{\text {init }} \sqsubseteq R\left(\eta, \kappa_{I}\right)\left(n_{0}, \tau_{\text {init }}\right) .
$$

By the definition of $R$,

$$
R\left(\eta, \kappa_{I}\right)\left(n_{0}, \tau_{\text {init }}\right)=\prod\left\{\kappa_{I}\left(n_{0}, \pi\right) \mid \tau_{\text {init }} \sqsubseteq \eta\left(\operatorname{method}\left(n_{0}\right), \pi\right)\right\} .
$$

Hence, it suffices to show that for every $\pi$,

$$
\tau_{\text {init }} \sqsubseteq \eta\left(\operatorname{method}\left(n_{0}\right), \pi\right) \Longrightarrow \tau_{\text {init }} \sqsubseteq \kappa_{I}\left(n_{0}, \pi\right) .
$$

By the assumption of the lemma, $\tau_{\text {init }} \sqsubseteq \eta\left(\operatorname{method}\left(n_{0}\right), \pi\right)$ can happen only if $\pi=m_{\text {main }}$. Furthermore, $\kappa_{I}\left(n_{0}, m_{\text {main }}\right)=\tau_{\text {init }}$. The desired inequality, hence, follows.

A translation $\eta$ is consistent with an $\infty$-CFA annotation $\kappa$ if the following two conditions hold:

1. For all $m \in \mathbf{M}, e \in \mathbf{E}$ and $\pi \in \mathbf{\Pi}$,

$$
\begin{aligned}
& (\operatorname{callEdge}(e) \wedge m \in \operatorname{calls}(\operatorname{stmt}(e), \kappa(\operatorname{origin}(e), \pi))) \\
& \Longrightarrow \kappa(\operatorname{origin}(e), \pi)=\eta(m, m \oplus e \oplus \pi) .
\end{aligned}
$$

2. For all $m \in \mathbf{M}$ and $\pi \in \boldsymbol{\Pi}$,

$$
\begin{aligned}
(\eta(m, \pi) \neq \perp & \left.\wedge m \neq m_{\text {main }}\right) \\
\Longrightarrow \exists e^{\prime}, \pi^{\prime} & : \pi=m \oplus e^{\prime} \oplus \pi^{\prime} \wedge \operatorname{callEdge}\left(e^{\prime}\right) \\
& \wedge m \in \operatorname{calls}\left(\operatorname{stmt}\left(e^{\prime}\right), \kappa\left(\operatorname{origin}\left(e^{\prime}\right), \pi^{\prime}\right)\right) .
\end{aligned}
$$


Lemma 9. For all translations $\eta$ and $\infty-C F A$ annotations $\kappa$, if $\eta$ is consistent with $\kappa$,

$$
F_{\mathrm{sba}}(R(\eta, \kappa)) \sqsubseteq R\left(\eta, F_{\mathrm{cfa}}(\kappa)\right) .
$$

Proof. Consider a translation $\eta$ and an $\infty$-CFA annotation $\kappa$ such that $\eta$ is consistent with $\kappa$. Pick $p, \tau$. We need to prove that

$$
F_{\text {sba }}(R(\eta, \kappa))(p, \tau) \sqsubseteq R\left(\eta, F_{\text {cfa }}(\kappa)\right)(p, \tau) .
$$

We discharge this proof obligation by doing the case analysis on $p$.

1. The first case is that $p$ is a node $n$ but not an entry node of any method. In this case,

$$
\begin{aligned}
& F_{\text {sba }}(R(\eta, \kappa))(n, \tau) \\
& =\bigsqcup\{R(\eta, \kappa)(e, \tau) \mid n=\operatorname{target}(e)\} \\
& =\bigsqcup\left\{\prod\{\kappa(e, \pi) \mid \tau \sqsubseteq \eta(\operatorname{method}(e), \pi)\} \mid n=\operatorname{target}(e)\right\} .
\end{aligned}
$$

Also,

$$
\begin{aligned}
& R\left(\eta, F_{\mathrm{cfa}}(\kappa)\right)(n, \tau) \\
& =\prod\left\{F_{\mathrm{cfa}}(\kappa)(n, \pi) \mid \tau \sqsubseteq \eta(\operatorname{method}(n), \pi)\right\} \\
& =\prod\{\bigsqcup\{\kappa(e, \pi) \mid n=\operatorname{target}(e)\} \mid \tau \sqsubseteq \eta(\operatorname{method}(n), \pi)\} .
\end{aligned}
$$

Hence, it suffices to prove the following: for all $e, \pi$, if $n=\operatorname{target}(e)$ and $\tau \sqsubseteq \eta(\operatorname{method}(n), \pi)$, we have that

$$
\begin{aligned}
& \prod\left\{\kappa\left(e, \pi^{\prime}\right) \mid \tau \sqsubseteq \eta\left(\operatorname{method}(e), \pi^{\prime}\right)\right\} \\
& \sqsubseteq \bigsqcup\left\{\kappa\left(e^{\prime}, \pi\right) \mid n=\operatorname{target}\left(e^{\prime}\right)\right\} .
\end{aligned}
$$

Since $\tau \sqsubseteq \eta(\operatorname{method}(n), \pi)$ and $n=\operatorname{target}(e)$,

$$
\tau \sqsubseteq \eta(\operatorname{method}(e), \pi) .
$$

Hence,

$$
\begin{aligned}
& \left(\prod\left\{\kappa\left(e, \pi^{\prime}\right) \mid \tau \sqsubseteq \eta\left(\operatorname{method}(e), \pi^{\prime}\right)\right\}\right) \sqsubseteq \kappa(e, \pi) \\
& \wedge \kappa(e, \pi) \sqsubseteq\left(\bigsqcup\left\{\kappa\left(e^{\prime}, \pi\right) \mid n=\operatorname{target}\left(e^{\prime}\right)\right\}\right) .
\end{aligned}
$$

This gives the desired inequality.

2. The next case is that $p$ is an entry node $\operatorname{entry}(m)$. Let $P\left(e, \tau_{1}\right)$ be the predicate that asserts the following:

$$
\begin{aligned}
& \text { callEdge }(e) \wedge \tau=R(\eta, \kappa)\left(\operatorname{origin}(e), \tau_{1}\right) \\
& \wedge m \in \operatorname{calls}(\operatorname{stmt}(e), \tau) .
\end{aligned}
$$


If we cannot find $e, \tau_{1}$ satisfying $P\left(e, \tau_{1}\right)$, then

$$
F_{\text {sba }}(R(\eta, \kappa))(\operatorname{entry}(m), \tau)=\perp,
$$

which implies the desired inequality in (23). Now assume that $P\left(e, \tau_{1}\right)$ holds for some $e$ and $\tau_{1}$. Since $m \in$ calls $(\operatorname{stm} t(e), \tau)$, by our requirement on calls, we have

$$
m \neq m_{\text {main }}
$$

Also, in this case,

$$
F_{\text {sba }}(R(\eta, \kappa))(\operatorname{entry}(m), \tau)=\tau \text {. }
$$

Meanwhile,

$$
\begin{aligned}
& R\left(\eta, F_{\mathrm{cfa}}(\kappa)\right)(\operatorname{entry}(m), \tau) \\
& =\prod\left\{F_{\mathrm{cfa}}(\kappa)(\operatorname{entry}(m), \pi) \mid \tau \sqsubseteq \eta(m, \pi)\right\}
\end{aligned}
$$

Thus, we can prove the desired inequality in 23 by showing that for all $\pi$, if $\tau \sqsubseteq \eta(m, \pi)$,

$$
\tau \sqsubseteq F_{\mathrm{cfa}}(\kappa)(\operatorname{entry}(m), \pi) .
$$

Since $m \in \operatorname{calls}(\operatorname{stmt}(e), \tau)$,

$$
\tau \neq \perp \text {. }
$$

Pick $\pi$ such that $\tau \sqsubseteq \eta(m, \pi)$. Then,

$$
\eta(m, \pi) \neq \perp .
$$

Since $m \neq m_{\text {main }}$, the consistency of $\eta$ with $\kappa$ implies

$$
\begin{aligned}
& \exists e^{\prime}, \pi^{\prime}: \pi=m \oplus e^{\prime} \oplus \pi^{\prime} \\
& \wedge \operatorname{callEdge}\left(e^{\prime}\right) \wedge m \in \operatorname{calls}\left(\operatorname{stmt}\left(e^{\prime}\right), \kappa\left(\operatorname{origin}\left(e^{\prime}\right), \pi^{\prime}\right)\right) .
\end{aligned}
$$

Again by the consistency of $\eta$ with $\kappa$, this entails

$$
\kappa\left(\operatorname{origin}\left(e^{\prime}\right), \pi^{\prime}\right)=\eta\left(m, m \oplus e^{\prime} \oplus \pi^{\prime}\right)=\eta(m, \pi) .
$$

From this fact, we derive that

$$
\begin{aligned}
F_{\text {cfa }}(\kappa)(\operatorname{entry}(m), \pi) & \sqsupseteq \kappa\left(\operatorname{origin}\left(e^{\prime}\right), \pi^{\prime}\right) \\
& =\eta(m, \pi) \sqsupseteq \tau .
\end{aligned}
$$

3. The third case is that $p$ is a non-call edge $e$ (i.e., $\neg$ callEdge $(e)$ ). In this case,

$$
\begin{aligned}
& F_{\text {sba }}(R(\eta, \kappa))(e, \tau) \\
& =\llbracket \operatorname{stmt}(e) \rrbracket(R(\eta, \kappa)(\operatorname{origin}(e), \tau)) \\
& =\llbracket \operatorname{stmt}(e) \rrbracket\left(\prod\{\kappa(\operatorname{origin}(e), \pi) \mid \tau \sqsubseteq \eta(\operatorname{method}(e), \pi)\}\right) .
\end{aligned}
$$


Also,

$$
\begin{aligned}
& R\left(\eta, F_{\mathrm{cfa}}(\kappa)\right)(e, \tau) \\
& =\prod\left\{F_{\mathrm{cfa}}(\kappa)(e, \pi) \mid \tau \sqsubseteq \eta(\operatorname{method}(e), \pi)\right\} \\
& =\prod\{\llbracket \operatorname{stmt}(e) \rrbracket(\kappa(\operatorname{origin}(e), \pi)) \mid \tau \sqsubseteq \eta(\operatorname{method}(e), \pi)\} .
\end{aligned}
$$

Hence, it suffices to prove that for all $\pi_{0}$, if

$$
\tau \sqsubseteq \eta\left(\operatorname{method}(e), \pi_{0}\right),
$$

then

$$
\begin{aligned}
& \llbracket \operatorname{stmt}(e) \rrbracket(\bigcap\{\kappa(\operatorname{origin}(e), \pi) \mid \tau \sqsubseteq \eta(\operatorname{origin}(e), \pi)\}) \\
& \sqsubseteq \llbracket \operatorname{stmt}(e) \rrbracket\left(\kappa\left(\operatorname{origin}(e), \pi_{0}\right)\right) .
\end{aligned}
$$

Pick such $\pi_{0}$. The desired inequality above holds because $\tau \sqsubseteq \eta\left(\operatorname{method}(e), \pi_{0}\right)$ and $\llbracket \operatorname{stmt}(e) \rrbracket$ is monotone.

4. The last case is that $p$ is a call edge $e$ (i.e., callEdge(e)). In this case,

$$
\begin{aligned}
& F_{\text {sba }}(R(\eta, \kappa))(e, \tau) \\
& =\bigsqcup\{R(\eta, \kappa)(\operatorname{exit}(m), R(\eta, \kappa)(\operatorname{origin}(e), \tau)) \mid \\
& m \in \operatorname{calls}(\operatorname{stmt}(e), R(\eta, \kappa)(\operatorname{origin}(e), \tau))\} .
\end{aligned}
$$

Also,

$$
\begin{aligned}
& R\left(\eta, F_{\mathrm{cfa}}(\kappa)\right)(e, \tau) \\
& =\prod\left\{F_{\mathrm{cfa}}(\kappa)(e, \pi) \mid \tau \sqsubseteq \eta(\operatorname{method}(e), \pi)\right\} .
\end{aligned}
$$

Hence, it is sufficient to prove that for all $m_{0}, \pi_{0}$, if

$$
\begin{aligned}
& \tau \sqsubseteq \eta\left(\operatorname{method}(e), \pi_{0}\right) \\
& \wedge m_{0} \in \operatorname{calls}(\operatorname{stmt}(e), R(\eta, \kappa)(\operatorname{origin}(e), \tau))
\end{aligned}
$$

then

$$
\begin{aligned}
& R(\eta, \kappa)\left(\operatorname{exit}\left(m_{0}\right), R(\eta, \kappa)(\operatorname{origin}(e), \tau)\right) \\
& \sqsubseteq F_{\mathrm{cfa}}(\kappa)\left(e, \pi_{0}\right) .
\end{aligned}
$$

Pick $m_{0}, \pi_{0}$ satisfying the assumptions in the above implication. Since $\tau \sqsubseteq$ $\eta\left(\operatorname{method}(e), \pi_{0}\right)$, we have

$$
\tau \sqsubseteq \eta\left(\operatorname{method}(\operatorname{origin}(e)), \pi_{0}\right),
$$

which implies that

$$
R(\eta, \kappa)(\operatorname{origin}(e), \tau) \sqsubseteq \kappa\left(\operatorname{origin}(e), \pi_{0}\right) .
$$

This gives

$$
m_{0} \in \operatorname{calls}\left(\operatorname{stmt}(e), \kappa\left(\operatorname{origin}(e), \pi_{0}\right)\right),
$$


because $m_{0} \in \operatorname{calls}(\operatorname{stmt}(e), R(\eta, \kappa)(\operatorname{origin}(e), \tau))$ and the function calls $(\operatorname{stmt}(e),-)$ is monotone. One consequence of (26) is that

$$
\kappa\left(\operatorname{exit}\left(m_{0}\right), m_{0} \oplus e \oplus \pi_{0}\right) \sqsubseteq F_{\mathrm{cfa}}(\kappa)\left(e, \pi_{0}\right) .
$$

Hence, to prove 25$]$, we only need to show that

$$
\begin{aligned}
& R(\eta, \kappa)\left(\operatorname{exit}\left(m_{0}\right), R(\eta, \kappa)(\operatorname{origin}(e), \tau)\right) \\
& \sqsubseteq \kappa\left(\operatorname{exit}\left(m_{0}\right), m_{0} \oplus e \oplus \pi_{0}\right) .
\end{aligned}
$$

Fortunately, by the definition of $R$, this proof obligation follows from a simpler property:

$$
R(\eta, \kappa)(\operatorname{origin}(e), \tau) \sqsubseteq \eta\left(m_{0}, m_{0} \oplus e \oplus \pi_{0}\right),
$$

which we prove as follows:

$$
\begin{aligned}
& \left(\tau \sqsubseteq \eta\left(\operatorname{origin}(e), \pi_{0}\right)\right. \\
& \left.\wedge \kappa\left(\operatorname{origin}(e), \pi_{0}\right)=\eta\left(m_{0}, m_{0} \oplus e \oplus \pi_{0}\right)\right) \\
& \Longrightarrow R(\eta, \kappa)(\operatorname{origin}(e), \tau) \sqsubseteq \eta\left(m_{0}, m_{0} \oplus e \oplus \pi_{0}\right) .
\end{aligned}
$$

This implication follows from the definition of $R$.

Recall that $\eta_{\mathrm{cfa}}=\lambda(m, \pi) . \kappa_{\mathrm{cfa}}(\operatorname{entry}(m), \pi)$.

Lemma 10. $\eta_{\text {cfa }}$ is consistent with $\kappa_{\text {cfa }}$ and

$$
\begin{aligned}
& \eta_{\mathrm{cfa}}\left(m_{\text {main }}, m_{\text {main }}\right)=\tau_{\text {init }} \\
& \wedge\left(\forall \pi: \pi \neq m_{\text {main }} \Longrightarrow \eta_{\text {cfa }}\left(m_{\text {main }}, \pi\right)=\perp\right) .
\end{aligned}
$$

Proof. Since $\kappa_{\mathrm{cfa}}$ is a fixpoint of $\lambda \kappa . \kappa_{I} \sqcup F_{\mathrm{cfa}}(\kappa)$, the following properties hold for $\eta_{\mathrm{cfa}}$ :

$$
\begin{aligned}
\eta_{\mathrm{cfa}}\left(m_{\text {main }}, m_{\text {main }}\right)= & \tau_{\text {init }} \\
\eta_{\mathrm{cfa}}(m, m \oplus e \oplus \pi)= & \text { if }(\operatorname{callEdge}(e) \wedge \\
& \left.m \in \operatorname{calls}\left(\operatorname{stmt}(e), \kappa_{\mathrm{cfa}}(\operatorname{origin}(e), \pi)\right)\right) \\
& \text { then } \kappa_{\mathrm{cfa}}(\operatorname{origin}(e), \pi) \text { else } \perp \\
\eta_{\mathrm{cfa}}(m, \pi)= & \perp \quad \text { (for all the other cases }) .
\end{aligned}
$$

Now it remains to show that satisfying the following three requirements:

1. For all $m \in \mathbf{M}, e \in \mathbf{E}$ and $\pi \in \boldsymbol{\Pi}$,

$$
\begin{aligned}
& \left(\operatorname{callEdge}(e) \wedge m \in \operatorname{calls}\left(\operatorname{stmt}(e), \kappa_{\mathrm{cfa}}(\operatorname{origin}(e), \pi)\right)\right) \\
& \Longrightarrow \kappa_{\mathrm{cfa}}(\operatorname{origin}(e), \pi)=\eta_{\mathrm{cfa}}(m, m \oplus e \oplus \pi) .
\end{aligned}
$$


2. For all $m \in \mathbf{M}$ and $\pi \in \boldsymbol{\Pi}$,

$$
\begin{aligned}
& \left(\eta_{\mathrm{cfa}}(m, \pi) \neq\right. \\
& \left.\Longrightarrow \exists e^{\prime}, \pi^{\prime}: \pi=m \oplus m_{\text {main }}\right) \\
& \quad \wedge m \in \operatorname{calls}\left(\operatorname{stmt}\left(e^{\prime}\right), \kappa_{\mathrm{cfa}}\left(\operatorname{origin}\left(e^{\prime}\right), \pi^{\prime}\right)\right)
\end{aligned}
$$

3. $\eta_{\mathrm{cfa}}\left(m_{\text {main }}, m_{\text {main }}\right)=\tau_{\text {init }}$ and

$$
\forall \pi: \pi \neq m_{\text {main }} \Longrightarrow \eta_{\text {cfa }}\left(m_{\text {main }}, \pi\right)=\perp \text {. }
$$

The first requirement is an immediate consequence of the properties of $\eta_{\text {cfa }}$ that we noticed in the beginning of the proof. To show the second requirement, consider $m \in \mathbf{M}$ and $\pi \in \boldsymbol{\Pi}$ such that

$$
\eta_{\mathrm{cfa}}(m, \pi) \neq \perp \wedge m \neq m_{\text {main }} .
$$

By the properties of $\eta_{\text {cfa }}$ in the beginning of this lemma, there exist $e^{\prime}$ and $\pi^{\prime}$ such that

$$
\begin{aligned}
& \pi=m \oplus e^{\prime} \oplus \pi^{\prime} \wedge \operatorname{callEdge}\left(e^{\prime}\right) \\
& \wedge m \in \operatorname{calls}\left(\operatorname{stmt}\left(e^{\prime}\right), \kappa_{\mathrm{cfa}}\left(\operatorname{origin}\left(e^{\prime}\right), \pi^{\prime}\right)\right) .
\end{aligned}
$$

We have just shown that the second requirement holds. For the final requirement, we first notice that $\eta_{\mathrm{cfa}}\left(m_{\text {main }}, m_{\text {main }}\right)=\tau_{\text {init }}$ by definition. To prove the remaining part of this final requirement, consider $\pi$ with $\pi \neq m_{\text {main }}$. For the sake of contradiction, suppose that

$$
\eta_{\mathrm{cfa}}\left(m_{\text {main }}, \pi\right) \neq \perp \text {. }
$$

Then, by the definition of $\eta_{\mathrm{cfa}}$, there exist $e^{\prime}, \pi^{\prime}$ such that

$$
\begin{aligned}
& \pi=m_{\text {main }} \oplus e^{\prime} \oplus \pi^{\prime} \wedge \text { callEdge }\left(e^{\prime}\right) \\
& \wedge m_{\text {main }} \in \operatorname{calls}\left(\operatorname{stmt}\left(e^{\prime}\right), \kappa_{\mathrm{cfa}}\left(\operatorname{origin}\left(e^{\prime}\right), \pi^{\prime}\right)\right) .
\end{aligned}
$$

This last conjunct contradicts our assumption that $m_{\text {main }}$ is not in the range of calls.

Let $\eta_{\text {cfa }}$ be the translation constructed from $\kappa_{\text {cfa }}$ by following the construction in the proof of Lemma 10 .

Proposition 2, $\sigma_{\text {sba }} \sqsubseteq R\left(\eta_{\mathrm{cfa}}, \kappa_{\mathrm{cfa}}\right)$.

Proof. Since $\kappa_{\text {cfa }}$ is the result of the $\infty$-CFA analysis,

$$
\kappa_{I} \sqcup F_{\text {cfa }}\left(\kappa_{\text {cfa }}\right) \sqsubseteq \kappa_{\text {cfa }} .
$$

As shown in Lemma 10. $\eta_{\text {cfa }}$ satisfies the assumptions of Lemma 8 . Furthermore, $R\left(\eta_{\mathrm{cfa}},-\right)$ is monotone. Hence,

$$
\sigma_{I} \sqsubseteq R\left(\eta_{\mathrm{cfa}}, \kappa_{I}\right) \sqsubseteq R\left(\eta_{\mathrm{cfa}}, \kappa_{\mathrm{cfa}}\right) .
$$


a Also, since $\eta_{\text {cfa }}$ is consistent with $\kappa_{\text {cfa }}$ and $F_{\text {cfa }}\left(\kappa_{\text {cfa }}\right) \sqsubseteq \kappa_{\text {cfa }}$, Lemma 9 and the monotonicity of $R\left(\eta_{\mathrm{cfa}},-\right)$ imply that

$$
F_{\text {sba }}\left(R\left(\eta_{\text {cfa }}, \kappa_{\text {cfa }}\right)\right) \sqsubseteq R\left(\eta_{\text {cfa }}, F_{\text {cfa }}\left(\kappa_{\text {cfa }}\right)\right) \sqsubseteq R\left(\eta_{\text {cfa }}, \kappa_{\text {cfa }}\right) .
$$

This means that $R\left(\eta_{\text {cfa }}, \kappa_{\text {cfa }}\right)$ is a pre-fixpoint of $\lambda \sigma . \sigma_{I} \sqcup F_{\text {sba }}(\sigma)$. In the rest of the proof, we will show that $R\left(\eta_{\mathrm{cfa}}, \kappa_{\mathrm{cfa}}\right)$ is monotone

$$
\forall p, \tau, \tau^{\prime}: \tau \sqsubseteq \tau^{\prime} \Longrightarrow R\left(\eta_{\mathrm{cfa}}, \kappa_{\mathrm{cfa}}\right)(\tau) \sqsubseteq R\left(\eta_{\mathrm{cfa}}, \kappa_{\mathrm{cfa}}\right)\left(\tau^{\prime}\right) .
$$

and

$$
\forall m \in \mathbf{M}: \forall \tau \in \boldsymbol{\Gamma}: \tau \sqsubseteq R\left(\eta_{\text {cfa }}, \kappa_{\text {cfa }}\right)(\operatorname{entry}(m), \tau) .
$$

Note that once these two are proved, Lemma 3 gives the desired $\sigma_{\mathrm{sba}} \sqsubseteq R\left(\eta_{\mathrm{cfa}}, \kappa_{\mathrm{cfa}}\right)$. The monotonicity of $R\left(\eta_{\mathrm{cfa}}, \kappa_{\mathrm{cfa}}\right)$ is immediate from the definition of $R$. To prove the other property, pick $m \in \mathbf{M}$ and $\tau \in \boldsymbol{\Gamma}$. We need to show that

$$
\tau \sqsubseteq R\left(\eta_{\mathrm{cfa}}, \kappa_{\mathrm{cfa}}\right)(\operatorname{entry}(m), \tau) .
$$

Since

$$
\begin{aligned}
& R\left(\eta_{\mathrm{cfa}}, \kappa_{\mathrm{cfa}}\right)(\operatorname{entry}(m), \tau) \\
& =\prod\left\{\kappa_{\mathrm{cfa}}(\operatorname{entry}(m), \pi) \mid \tau \sqsubseteq \eta_{\mathrm{cfa}}(m, \pi)\right\},
\end{aligned}
$$

our proof obligation can be discharged by showing that for every $\pi$,

$$
\eta_{\mathrm{cfa}}(m, \pi) \sqsubseteq \kappa_{\mathrm{cfa}}(\operatorname{entry}(m), \pi) .
$$

But $\eta_{\mathrm{cfa}}(m, \pi)=\kappa_{\mathrm{cfa}}(\operatorname{entry}(m), \pi)$ by definition.

\section{B.3 Step Three}

Proposition 3. $L\left(\eta_{\mathrm{cfa}}, \sigma_{\mathrm{sba}}\right) \sqsubseteq \kappa_{\mathrm{cfa}} \sqsubseteq L\left(\eta_{\mathrm{sba}}, \sigma_{\mathrm{sba}}\right)$.

Proof. By Proposition 2 ,

$$
\sigma_{\mathrm{sba}} \sqsubseteq R\left(\eta_{\mathrm{cfa}}, \kappa_{\mathrm{cfa}}\right) .
$$

By Lemma 1 .

$$
L\left(\eta_{\mathrm{cfa}}, \sigma_{\mathrm{sba}}\right) \sqsubseteq \kappa_{\mathrm{cfa}} .
$$

The other inequality follows from Proposition 1.

\section{B.4 Step Four}

Lemma 11. $\eta_{\text {cfa }}=\eta_{\text {sba }}$. 
Proof. Recall that $\eta_{\text {sba }}$ is defined as the least upper bound of a sequence $\left\{\eta_{n}\right\}_{n \geq 0}$ :

$$
\eta_{\text {sba }}(m, \pi)=\bigsqcup\left\{\eta_{n}(m, \pi) \mid n \geq 0\right\}
$$

where $\eta_{0}$ is the constant map $\lambda(m, \pi) . \perp$ and $\eta_{n+1}$ is defined by:

$$
\begin{aligned}
& \eta_{n+1}\left(m_{\text {main }}, m_{\text {main }}\right)=\tau_{\text {init }} \\
& \eta_{n+1}(m, m \oplus e \oplus \pi)= \\
& \quad \text { if }(\operatorname{callEdge}(e) \\
& \left.\quad \wedge m \in \operatorname{calls}\left(\operatorname{stmt}(e), \sigma_{\text {sba }}\left(\operatorname{origin}(e), \eta_{n}(\operatorname{method}(e), \pi)\right)\right)\right) \\
& \quad \text { then } \sigma_{\text {sba }}\left(\operatorname{origin}(e), \eta_{n}(\operatorname{method}(e), \pi)\right) \text { else } \perp \\
& \eta_{n+1}(p, \pi)=\perp \quad(\text { for all the other cases }) .
\end{aligned}
$$

Also, the following properties of $\eta_{\text {cfa }}$ hold because $\kappa_{\text {cfa }}$ is a fixpoint of $\lambda \kappa . \kappa_{I} \sqcup$ $F_{\mathrm{cfa}}(\kappa)$ and $\eta_{\mathrm{cfa}}=\lambda(m, \pi) . \kappa_{\mathrm{cfa}}(\operatorname{entry}(m), \pi)$ :

$$
\begin{aligned}
& \begin{array}{l}
\eta_{\mathrm{cfa}}\left(m_{\text {main }}, m_{\text {main }}\right)= \\
\eta_{\text {cfa }}(m, m \oplus e \oplus \pi)= \\
\quad \text { if }(\operatorname{callEdge}(e) \wedge \\
\left.\quad m \in \operatorname{calls}\left(\operatorname{stmt}(e), \kappa_{\mathrm{cfa}}(\operatorname{origin}(e), \pi)\right)\right) \\
\quad \text { then } \kappa_{\mathrm{cfa}}(\operatorname{origin}(e), \pi) \text { else } \perp
\end{array} \\
& \begin{array}{c}
\left.\eta_{\mathrm{cfa}}(p, \pi)=\perp \quad \text { (for all the other cases }\right) .
\end{array}
\end{aligned}
$$

Another thing is the following fact that we already have shown while proving Lemma 2 .

$$
\begin{aligned}
& \forall n, m, \pi: \eta_{n}(m, \pi) \neq \perp \Longrightarrow \\
&\left(\forall k \geq n: \eta_{k}(m, \pi)=\eta_{n}(m, \pi)\right) .
\end{aligned}
$$

We will prove the following two properties:

1. $\forall n, m, \pi: \eta_{n}(m, \pi) \neq \perp \Longrightarrow \eta_{n}(m, \pi)=\eta_{\mathrm{cfa}}(m, \pi)$.

2. $\forall m, \pi: \eta_{\text {sba }}(m, \pi)=\perp \Longrightarrow \eta_{\text {cfa }}(m, \pi)=\perp$.

We show the first property by induction on $n$.

1. Base case $n=0$ : The property holds in this case because $\eta_{n}(m, \pi)=\perp$ for all $m$ and $\pi$.

2. Inductive case $n>0$ : Pick $m, \pi$ such that $\eta_{n}(m, \pi) \neq \perp$. By the definition of $\eta_{n}$, this can happen if $\pi=m_{\text {main }} \wedge m=m_{\text {main }}$ or there exist $e, \pi^{\prime}$ such that

$$
\begin{aligned}
& \pi=m \oplus e \oplus \pi^{\prime} \wedge \text { callEdge }(e) \\
& \wedge m \in \operatorname{calls}\left(\operatorname{stmt}(e), \sigma_{\text {sba }}\left(\operatorname{origin}(e), \eta_{n-1}\left(\operatorname{method}(e), \pi^{\prime}\right)\right)\right) .
\end{aligned}
$$

If $\pi=m_{\text {main }}$ and $m=m_{\text {main }}$, the desired equality holds as shown below:

$$
\eta_{\text {sba }}(m, \pi)=\tau_{\text {init }}=\eta_{\mathrm{cfa}}(m, \pi) .
$$


To handle the other case, assume the existence of $e, \pi^{\prime}$ satisfying the condition described above. Then,

$$
\begin{aligned}
& \sigma_{\text {sba }}\left(\operatorname{origin}(e), \eta_{n-1}\left(\operatorname{method}(e), \pi^{\prime}\right)\right) \neq \perp \\
& \wedge \eta_{n}\left(p, m \oplus e \oplus \pi^{\prime}\right)=\sigma_{\text {sba }}\left(\operatorname{origin}(e), \eta_{n-1}\left(\operatorname{method}(e), \pi^{\prime}\right)\right) .
\end{aligned}
$$

Since $\sigma_{\text {sba }}$ is strict (Lemma 4), the first conjunct above implies $\eta_{n-1}\left(\operatorname{method}(e), \pi^{\prime}\right) \neq$ $\perp$. Because of induction hypothesis, this gives

$$
\eta_{n-1}\left(\operatorname{method}(e), \pi^{\prime}\right)=\eta_{\mathrm{cfa}}\left(\operatorname{method}(e), \pi^{\prime}\right)
$$

Also, by (27) and the definition of $\eta_{\text {sba }}$,

$$
\eta_{\text {sba }}\left(\operatorname{method}(e), \pi^{\prime}\right)=\eta_{n-1}\left(\operatorname{method}(e), \pi^{\prime}\right) .
$$

Meanwhile, by Proposition 3 .

$$
\begin{aligned}
& L\left(\eta_{\mathrm{cfa}}, \sigma_{\mathrm{sba}}\right)\left(\operatorname{origin}(e), \pi^{\prime}\right) \sqsubseteq \kappa_{\mathrm{cfa}}\left(\operatorname{origin}(e), \pi^{\prime}\right) \\
& \wedge \kappa_{\mathrm{cfa}}\left(\operatorname{origin}(e), \pi^{\prime}\right) \sqsubseteq L\left(\eta_{\mathrm{sba}}, \sigma_{\mathrm{sba}}\right)\left(\operatorname{origin}(e), \pi^{\prime}\right) .
\end{aligned}
$$

Because of (28) and $(29)$, this implies that

$$
\sigma_{\mathrm{sba}}\left(\operatorname{origin}(e), \eta_{n-1}\left(\operatorname{method}(e), \pi^{\prime}\right)\right)=\kappa_{\mathrm{cfa}}\left(\operatorname{origin}(e), \pi^{\prime}\right),
$$

which gives

$$
m \in \operatorname{calls}\left(\operatorname{stmt}(e), \kappa_{\mathrm{cfa}}\left(\operatorname{origin}(e), \pi^{\prime}\right)\right) .
$$

Using what we have shown so far, we complete the proof of this inductive case:

$$
\begin{aligned}
\eta_{n}\left(m, m \oplus e \oplus \pi^{\prime}\right) & =\sigma_{\mathrm{sba}}\left(\operatorname{method}(e), \eta_{n-1}\left(\operatorname{method}(e), \pi^{\prime}\right)\right) \\
& =\kappa_{\mathrm{cfa}}\left(\operatorname{method}(e), \pi^{\prime}\right) \\
& =\eta_{\mathrm{cfa}}\left(m, m \oplus e \oplus \pi^{\prime}\right)
\end{aligned}
$$

The second equality is from (30), and the third holds because of (31) and our choice of $e$ and $p$.

It remains to show that

$$
\forall m, \pi: \eta_{\text {sba }}(m, \pi)=\perp \Longrightarrow \eta_{\text {cfa }}(m, \pi)=\perp .
$$

Pick $m, \pi$ such that $\eta_{\text {sba }}(m, \pi)=\perp$. We prove it by induction on the length of $\pi$.

1. Base case $|\pi| \leq 1$. For all $\pi$ with length at most $1, \eta_{\text {cfa }}$ and all of $\eta_{n}$ 's have the same definition. Hence, $\eta_{\mathrm{cfa}}(m, \pi)=\perp$. 
2. Inductive case $|\pi| \geq 2$. Suppose that $\eta_{\text {cfa }}(m, \pi) \neq \perp$. In order for this to happen, there should exist $e, \pi^{\prime}$ such that

$$
\begin{aligned}
& \pi=m \oplus e \oplus \pi^{\prime} \wedge \text { callEdge }(e) \\
& \wedge m \in \operatorname{calls}\left(\operatorname{stmt}(e), \kappa_{\mathrm{cfa}}\left(\operatorname{origin}(e), \pi^{\prime}\right)\right) \\
& \wedge \kappa_{\mathrm{cfa}}\left(\operatorname{origin}(e), \pi^{\prime}\right) \neq \perp .
\end{aligned}
$$

By the induction hypothesis and the first property of $\eta_{\text {sba }}$ on the non- $\perp$ case,

$$
\eta_{\mathrm{sba}}\left(\operatorname{method}(e), \pi^{\prime}\right)=\eta_{\mathrm{cfa}}\left(\operatorname{method}(e), \pi^{\prime}\right) .
$$

Meanwhile, by Proposition 3 .

$$
\begin{aligned}
& L\left(\eta_{\mathrm{cfa}}, \sigma_{\mathrm{sba}}\right)\left(\operatorname{origin}(e), \pi^{\prime}\right) \sqsubseteq \kappa_{\mathrm{cfa}}\left(\operatorname{origin}(e), \pi^{\prime}\right) \\
& \wedge \kappa_{\mathrm{cfa}}\left(\operatorname{origin}(e), \pi^{\prime}\right) \sqsubseteq L\left(\eta_{\mathrm{sba}}, \sigma_{\mathrm{sba}}\right)\left(\operatorname{origin}(e), \pi^{\prime}\right) .
\end{aligned}
$$

From these inequalities and the equality in 32 follows that

$$
\sigma_{\mathrm{sba}}\left(\operatorname{origin}(e), \eta_{\mathrm{sba}}\left(\operatorname{method}(e), \pi^{\prime}\right)\right)=\kappa_{\mathrm{cfa}}\left(\operatorname{origin}(e), \pi^{\prime}\right) .
$$

Hence,

$$
m \in \operatorname{calls}\left(\operatorname{stmt}(e), \sigma_{\mathrm{sba}}\left(\operatorname{origin}(e), \eta_{\mathrm{sba}}\left(\operatorname{method}(e), \pi^{\prime}\right)\right)\right) .
$$

This implies that

$$
\eta_{\text {sba }}(m, \pi)=\sigma_{\text {sba }}\left(\operatorname{origin}(e), \eta_{\text {sba }}\left(\operatorname{method}(e), \pi^{\prime}\right)\right) .
$$

Hence, $\eta_{\text {sba }}(m, \pi) \neq \perp$, which contradicts our assumption.

\section{B.5 Putting It All Together}

Theorem 2 . (Correspondence) $L\left(\eta_{\text {cfa }}, \sigma_{\text {sba }}\right)=\kappa_{\text {cfa }}$.

Proof. By Proposition 3 ,

$$
L\left(\eta_{\mathrm{cfa}}, \sigma_{\mathrm{sba}}\right) \sqsubseteq \kappa_{\mathrm{cfa}} \sqsubseteq L\left(\eta_{\mathrm{sba}}, \sigma_{\mathrm{sba}}\right) .
$$

By Lemma 11, $\eta_{\mathrm{cfa}}=\eta_{\mathrm{sba}}$. Hence, the above inequalities imply that

$$
L\left(\eta_{\mathrm{cfa}}, \sigma_{\mathrm{sba}}\right)=\kappa_{\mathrm{cfa}}=L\left(\eta_{\mathrm{sba}}, \sigma_{\mathrm{sba}}\right) .
$$

